\title{
Notch Signaling Commits Mesoderm to the Cardiac Lineage
}

\author{
Evan S. Bardot ${ }^{1,2,3}$, Bharati Jadhav ${ }^{2,4}$, Nadeera Wickramasinghe ${ }^{1,2,3}$, Amélie Rezza ${ }^{1,2,3}$, Michael \\ Rend1 ${ }^{1,2,3}$, Andrew J. Sharp ${ }^{2,4}$, and Nicole C. Dubois ${ }^{1,2,3,5}$ \\ 1Department of Cell, Developmental, and Regenerative Biology, \\ ${ }^{2}$ Mindich Child Health and Development Institute, \\ ${ }^{3}$ Black Family Stem Cell Institute, \\ ${ }^{4}$ Department of Genetics and Genomic Sciences, \\ Icahn School of Medicine at Mount Sinai, New York, NY 10029, USA
}

${ }^{5}$ Correspondence: Icahn School of Medicine at Mount Sinai, Hess Building 8-112, Box 1040; 1470

Madison Ave, New York, NY 10029, USA

E-mail address: nicole.dubois@mssm.edu

Key words: cardiac mesoderm, cardiomyocyte, cell fate specification, Notch, Wnt, hPSC differentiation

Running title: Notch during cardiac mesoderm commitment

\section{Summary statement}

Early fate decisions are dictated by the embryonic signaling environment. We show that Notch signaling is active during early mouse development and that activating Notch in human cardiac mesoderm enhances cardiomyocyte differentiation efficiency. 


\section{Abstract}

During development multiple progenitor populations contribute to the formation of the four-chambered heart and its diverse lineages. However, the underlying mechanisms that result in the specification of these progenitor populations are not yet fully understood. We have previously identified a population of cells that gives rise selectively to the heart ventricles but not the atria. Here, we have used this knowledge to transcriptionally profile subsets of cardiac mesoderm from the mouse embryo and have identified an enrichment for Notch signaling components in ventricular progenitors. Using directed differentiation of human pluripotent stem cells, we next investigated the role of Notch in cardiac mesoderm specification in a temporally controlled manner. We show that transient Notch induction in mesoderm increases cardiomyocyte differentiation efficiency, while maintaining cardiomyocytes in an immature state. Finally, our data suggest that Notch interacts with WNT to enhance commitment to the cardiac lineage. Overall, our findings support the notion that key signaling events during early heart development are critical for proper lineage specification and provide evidence for early roles of Notch and WNT during mouse and human heart development.

\section{Introduction}

An anatomically and functionally normal heart is the result of a coordinated series of specification events and cell movements that begin as early as gastrulation (Meilhac et al., 2014; Vincent and Buckingham, 2010). Fate mapping of the early mouse embryo revealed the organized manner in which cells of the epiblast migrate through the primitive streak (PS) to become members of the separate germ layers (Lawson et al., 1991). The consistency of these movements strongly suggests that the location and timing of ingression through the PS are determinants of cell fate. Notably, the earliest cells to ingress migrate across the embryo to become anterior structures, with those that do so later taking up more posterior positions (Lawson et al., 1991). This pattern is consistent in zebrafish, with the first cells to gastrulate migrating to the future heart region ahead of those that ingress later (Keegan, 2004). A series of experiments in avian embryos support the findings from mice, which assigned the prospective cardiogenic region of the presumptive PS and further delineate the time sensitivity of gastrulation movements in chick heart development (Garcia-Martinez and Schoenwolf, 1993; Inagaki et al., 1993). Importantly, epiblast cells fated to contribute to the heart are not committed to the cardiac lineage until shortly after they ingress through the PS, suggesting that lineage commitment occurs after gastrulation (Inagaki et al., 1993; Tam et al., 1997; Yutzey and Bader, 1995).

Recent studies using genetic lineage tracing approaches have greatly advanced our understanding of these earliest events during heart development. Studies using Mesp1-Cre lineage tracing have further solidified the mechanisms proposed from fate mapping data (Devine et al., 2014; Lescroart et al., 2014; Saga et al., 1999). Specifically, the first cells to express Mesp1 contribute to derivatives of the first heart field, primarily the left ventricle. As more cells express Mesp1 and become specified to the cardiac lineage, they contribute to the heart from the right ventricle, atria, and finally outflow tract (Lescroart et al., 2014). Previous work from our lab revealed the presence of a population of cells that transiently expresses Foxa2 during gastrulation and gives rise specifically to ventricular cardiovascular cells of the heart, likely representing cells that ingress and express Mesp1 early (Bardot et al., 2017b). The striking similarity between the mouse, zebrafish, and chick models suggest conservation of the regional organization and sequence of early events 
during heart morphogenesis. The tools developed in these studies enable us to understand the early steps of heart development with increased resolution, at the time when heart progenitors are becoming committed to the cardiac lineage.

While patterns of early migration are well established, the heterogeneity within the cardiac progenitor pool and the signaling environments to which early and late-specified cells are exposed are not yet well defined. Recent work has dissected the transcriptional landscape within the Mesp1-expressing cardiac progenitor pool (Lescroart et al., 2018; Scialdone et al., 2016). These analyses showed that cardiac progenitors are largely homogenous through E7.25. Between E7.25 and $\mathrm{E} 7.5$, as cells progress along the differentiation trajectory, two groups can be identified reflecting the first and second heart field lineages (Lescroart et al., 2018). What remains unclear is how exposure to different signaling environments affects the differentiation trajectories of these progenitor populations.

Here, we have isolated several distinct mesoderm and endoderm populations from the mid gastrulation mouse embryo and utilized comparative transcriptome analysis to identify key regulatory pathways characteristic of each subpopulation. Specifically, we identified Notch signaling as a pathway for in vivo cardiac mesoderm specification, and showed that transient induction of Notch signaling in human pluripotent stem cell (hP$\mathrm{SC}$ )-derived mesoderm enhances cardiomyocyte differentiation. Cardiomyocytes from Notch-induced cultures were less mature than their control counterparts and lacked a clear atrial vs. ventricular identity. We show that the increase in cardiomyocyte generation is a mesoderm specific effect, likely through interaction with other pathways important for mesoderm specification. We further validated this hypothesis through the modulation of
Notch and WNT signaling in hPSC differentiation to cardiomyocytes, demonstrating that Notch signaling activation is sufficient for commitment to the cardiac lineage and necessary for differentiation in the absence of WNT inhibition. These data help us to better understand the earliest steps during cardiac commitment and highlight the similarities between the mouse and human differentiation programs.

\section{Results}

\section{Identification and characterization of distinct mesoderm subpopulations in the gastrulating mouse embryo}

We have previously used lineage-tracing with Foxa2Cre to identify a cardiac progenitor population that gives rise selectively to the ventricles of the heart, but not the atria (Sup. Fig. 1a) (Bardot et al., 2017b). To label the ventricular lineage during embryonic development, we crossed Foxa2Cre mice with ROSA26-mTmG reporter mice, which express membrane-tethered Tomato in cells unexposed to Cre, but express membrane-tethered GFP after Cre recombination. Foxa2 lineage-traced cells ( $\mathrm{mGFP}+$ ) can be identified within the migratory mesodermal wings shortly after ingressing through the primitive streak (Sup. Fig. 1b), and later in the cardiogenic region below the head folds at the anterior side of the embryo (Sup. Fig. 1c). Foxa2 lineage-traced cells are restricted to a subdomain of the cardiac crescent and localize to the ventricular regions of the heart at all stages examined (Sup. Fig. 1d-g). This approach therefore provides us the unique opportunity to examine the ventricular and atrial lineages at any stage during development, starting as early as during gastrulation.

To characterize the earliest stage of such lineage specification we focused on mid gastru- 

available under aCC-BY-NC-ND 4.0 International license.
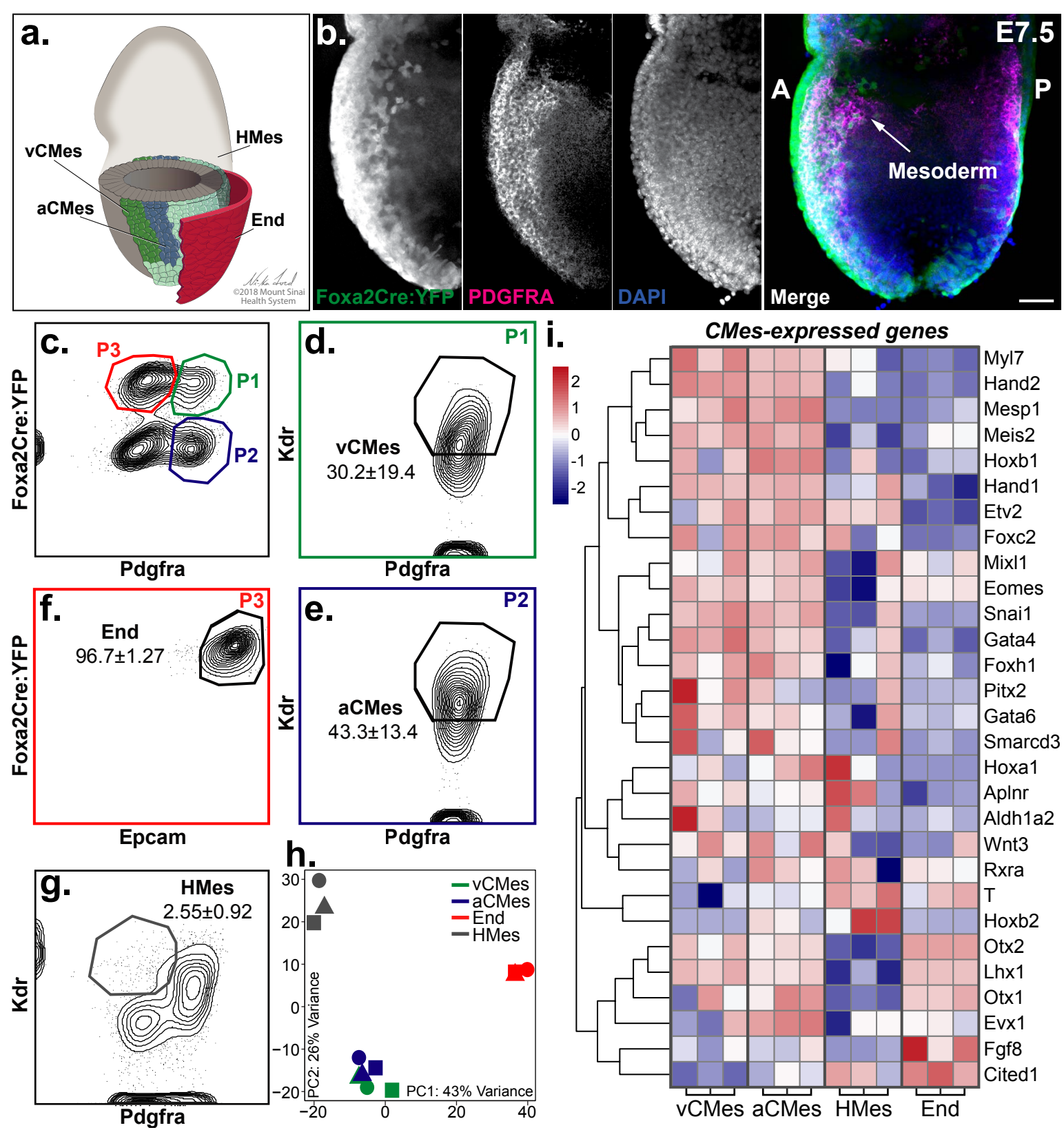

Figure 1: Isolation and gene expression profiling of early progenitor populations from mouse embryos. (a) Schematic of E7.5 embryo and populations of interest, including ventricular and non-ventricular cardiac mesoderm, endoderm, and hematopoietic mesoderm. (b) WMIF analysis of an E7.5 Foxa2Cre:YFP embryo with antibodies against YFP and PDGFRA. (c) Isolation strategy for fluorescence activated cell sorting from pooled Foxa2Cre:YFP embryos. Cells are first separated by expression of PDGFRA and YFP. (d) Ventricular (YFP+, P1) and (e) non-ventricular (YFP-, P2) CMes are identified by expression of PDGFRA and KDR. (f) Endoderm (YFP+, P3) is identified by expression of EPCAM. (g) Hematopoietic mesoderm (Hem. Mes.) is isolated based on single positivity for KDR. (h) Principal component analysis of whole-transcriptome RNA-sequencing data from isolated populations. (i) Expression z-scores of selected genes known to be expressed in Mesp1+ cardiac progenitors (genes taken from (Lescroart et al., 2014)). A, anterior; P, posterior. Scale bar is $100 \mu \mathrm{m}$.

lation (E7.25), shortly after cardiac mesoderm is formed. At this point during development, ventricular cardiac mesoderm (vCMes) migrates anteriorly, just ahead of the atrial cardiac mesoderm (aCMes) (Fig. 1a). Whole mount immunofluorescence (WMIF) analysis of Foxa2Cre:ROSA26-YFP embryos revealed Foxa2Cre:YFP+ cells that express the mesodermal marker PDGFRA as well as Foxa2Cre:YFP-PDGFRA+ cells (Fig. 1b). To elucidate the mechanisms at play as the different mesoderm populations migrate to the anterior side of the embryo and become progressively specified, we FACS purified cells from pooled, stage-matched embryos of the same litter for whole transcriptome 


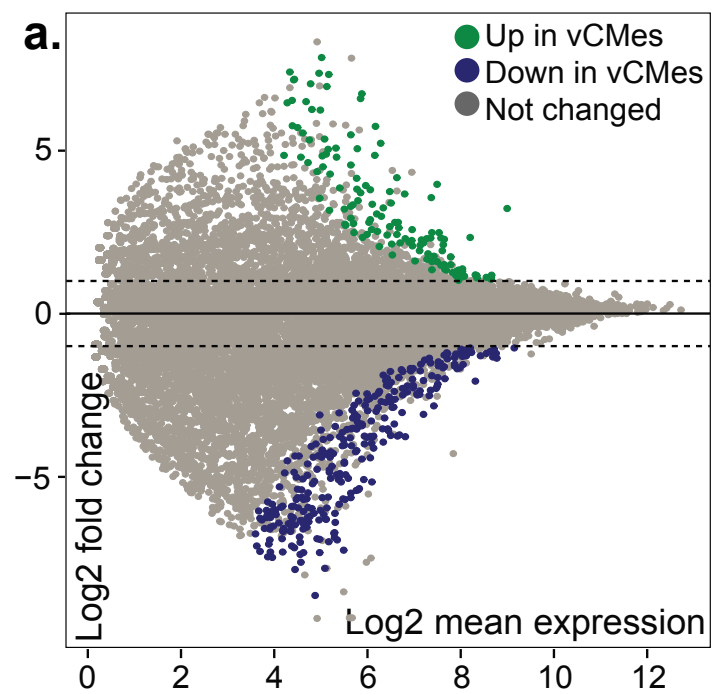

C.

\begin{tabular}{|c|c|c|c|c|}
\hline Sample & KEGG Term & Genes & p-value & $\begin{array}{c}\text { Fold } \\
\text { Enrichment }\end{array}$ \\
\hline $\begin{array}{c}\text { E7.25 in vivo } \\
\text { vCMes }\end{array}$ & $\begin{array}{c}\text { motch signaling } \\
\text { pathway }\end{array}$ & $\begin{array}{c}\text { Psen2, Jag1, } \\
\text { Mam/3 }\end{array}$ & 0.029 & 10.9 \\
\hline $\begin{array}{c}\text { Day 4 in vitro } \\
\text { vCMes }\end{array}$ & $\begin{array}{c}\text { Notch signaling } \\
\text { pathway }\end{array}$ & $\begin{array}{c}\text { Psen2, Kat2b, } \\
\text { Mam/2, Mam/3 }\end{array}$ & 0.049 & 4.77 \\
\hline
\end{tabular}

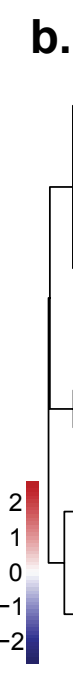

b.

Fold
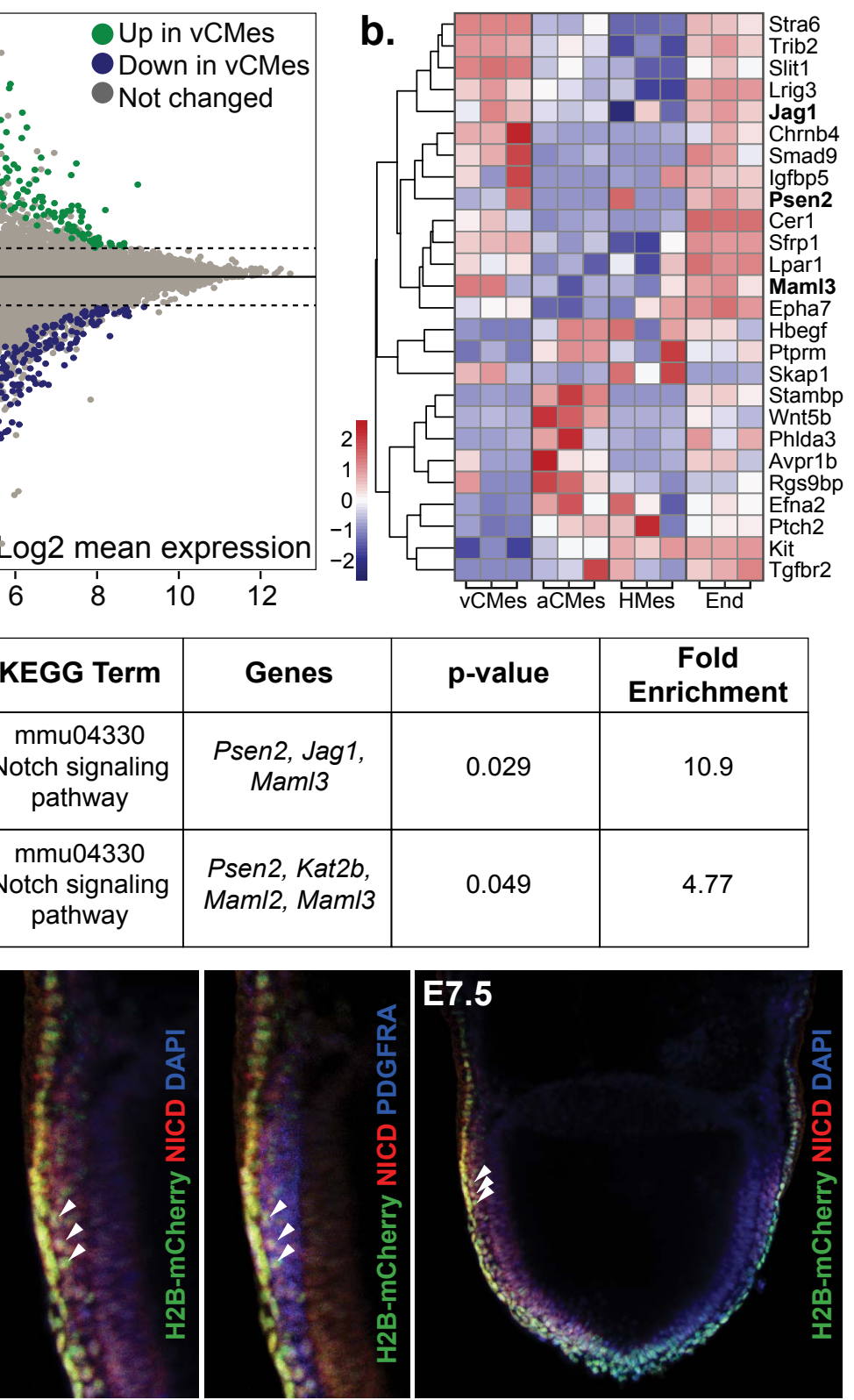

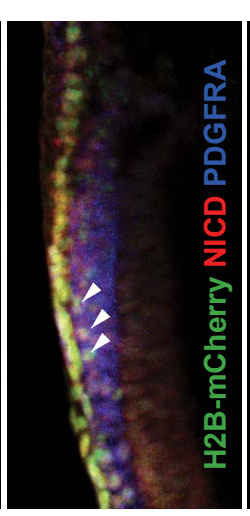

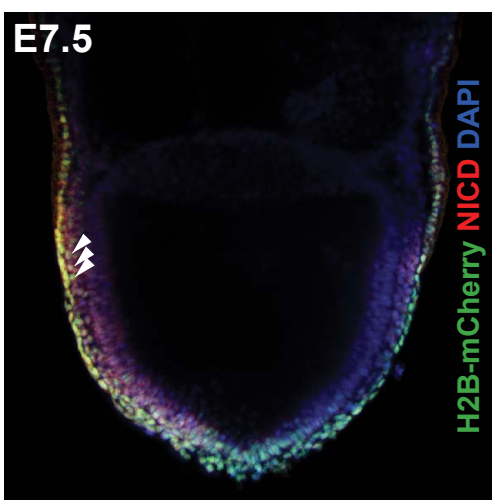

Figure 2: Gene expression analysis reveals a potential role for Notch signaling during cardiac mesoderm differentiation. (a) MA plot showing genes differentially expressed (FDR 5\%, fold change $>1.5$ ) between in vivo YFP+ CMes and YFP- CMes.

(b) Expression z-scores for differentially expressed signaling pathway components. (c) KEGG pathway analysis for genes enriched in YFP+ CMes in vivo, and comparison with in vitro cardiac mesoderm data. (d) WMIF analysis of an E7.5 Foxa2Cre:H2B-mCherry embryo with antibodies against mCherry, NICD, and PDGFRA. Embryo is imaged en face to show bilateral mesoderm.

RNA sequencing (Fig. 1c). We used antibodies against the cell surface markers PDGFRA and KDR to isolate CMes from the Foxa2Cre:YFP+ and Foxa2Cre:YFP- lineages, representing early migrating vCMes and late migrating aCMes, respectively (Fig. 1d, e) (Bardot et al., 2017b; Kattman et al., 2011). We also isolated hematopoietic mesoderm (HMes, KDRhigh) and endoderm cells (End, Foxa2Cre:YFP+, EPCAM+) as a non-mesodermal reference (Fig. 1f, g). From pooled litters, we obtained on average 273 vCMes, 840 aCMes, 936 HMes, and 1536 End cells (Sup. Fig. 2a). The difference in the number of each CMes subset recovered reflects their proportion of the total CMes population (Sup. Fig. 2b).

Three biological replicates passed a number of QC steps, including hierarchical clustering after batch correction for hidden covariates (Sup. Fig. 2c, d). Faithful expression of signature genes for cardiac mesoderm, endoderm, and hematopoietic mesoderm confirmed the expected overall identity of the isolated cell populations (Sup. Fig 2e). Principal component analysis (PCA) separated the mesoderm and endoderm lineages (PC1, $43 \%$ variance captured), and the cardiac from non-cardiac samples (PC2, 26\% variance captured) (Fig. 1h). As expected, PCA shows a high similarity between the different CMes populations 
at this stage, as these share the core cardiac mesoderm gene signature (Fig. 1h). Both vCMes and aCMes samples expressed genes known to be enriched in Mesp1+ cardiac progenitors (Fig. 1i) (Lescroart et al., 2014).

We have previously performed gene expression analysis on mouse embryonic stem cell-derived CMes populations representing the Foxa2+ and Foxa2- progeny in vitro (Bardot et al., 2017b). PCA comparing the in vitro populations with their in vivo counterparts initially showed large differences between the two systems, with PC1 separating the two datasets (Sup. Fig. 3a). Analysis of additional components restored the biological lineage relationships observed previously. Specifically, PC2 separated the mesoderm and endoderm populations, and PC3 isolated the cardiac mesoderm from non-cardiac lineages (Sup. Fig. 3a, b). Direct comparison of PC2 and PC3 resembled the relationships seen when analyzing either in vitro or in vivo samples individually (Sup. Fig. 3c). Examining the genes driving the variance for each PC reveals that characteristic marker genes for mesoderm, endoderm, or cardiac mesoderm account for the separation seen in PC2 and PC3 (Sup. Fig. 3d).

To elucidate differences between the $\mathrm{vC}$ Mes and aCMes populations we first performed differential gene expression analysis. We found 416 differentially expressed genes (FDR $<5 \%$, fold change>1.5), with 129 and 287 genes enriched in the vCMes and aCMes populations, respectively (Fig. 2a). We focused our analysis on signaling pathways since we hypothesize that the signaling environment at this stage of development will affect cell fate decisions. Of the top 200 most upand down-regulated genes, 26 have been shown to have signaling roles (Fig. 2b). Further analysis using KEGG pathway datasets revealed Notch signaling to be the only statistically enriched path- way within the vCMes population (Fig. 2c). This is consistent with our previous in vitro CMes gene expression data, illustrating the comparability of the in vivo and in vitro systems at similar stages of differentiation (Fig. 2c) (Bardot et al., 2017b). In addition, active Notch signaling could be observed in E7.5 mouse embryos, as detected by whole mount immunofluorescence for the Notch Intracellular Domain (NICD) (Fig. 2d). NICD+ nuclei are found within the PDGFRA+ mesoderm and can be seen in both the ventricular (Foxa2Cre:H2B-mCherry+) and atrial (Foxa2Cre:H2B-mCherry-) lineages. Overall, these analyses show that Notch signaling is active within the cardiac mesoderm in vivo and suggest a temporal gradient in the induction of this pathway during CMes differentiation.

\section{Activation of Notch signaling enhances car- diomyocyte differentiation from human plurip- otent stem cells}

We have identified Notch pathway genes as being differentially expressed in cardiac mesoderm populations in mice, suggesting a potential role during early differentiation. To dissect the role of Notch during cardiac mesoderm commitment and differentiation in a temporally-controlled manner, we turned to the well-established human pluripotent stem cell (hPSC) differentiation system. In this model, hPSCs are differentiated in the presence of signaling molecules that recapitulate the in vivo signaling environment (Kattman et al., 2011; Yang et al., 2008). In doing so, cells are guided along a normal developmental trajectory first exiting pluripotency, being patterned into mesoderm and specified to cardiac mesoderm, becoming committed to the cardiac lineage, and finally differentiating into functional cardiomyocytes (Fig. 3a) (Calderon et al., 2016; Kattman et al., 2011; Lian et al., 2013; Yang et al., 2008). To specifically manipulate Notch signaling during this process, we used 

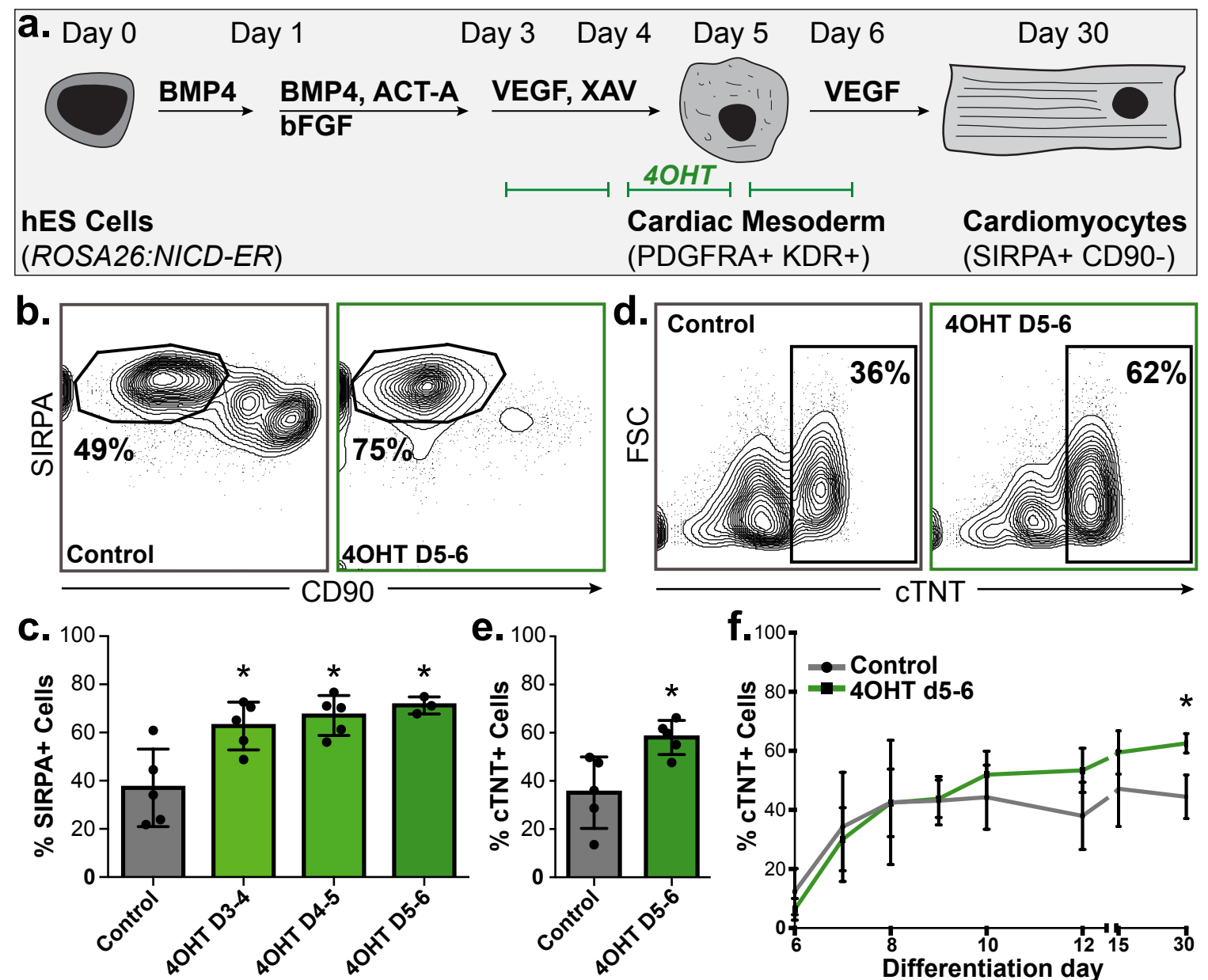

Figure 3: Activation of Notch signaling enhances cardiomyocyte differentiation from human pluripotent stem cells. (a) Schematic of ROSA26:NICD-ER hPSC-cardiomyocyte differentiation and Notch induction times. (b) Flow cytometric analysis with antibodies against SIRPA and CD90 to measure cardiomyocyte (SIRPA+CD90-) differentiation efficiency at day 30 in control cultures (left) or after 4OHT treatment from day 5-6 (right) (c) Quantification of data in (b). (d) Flow cytometric analysis of day 30 control (left) or Notch-induced (right) cultures with antibodies against cTNT. (e) Quantification of data in (d). (f) Flow cytometric measurement of cTNT expression over the course of differentiation $(n=3$ differentiations).

an hPSC line that expresses a Notch intracellular domain-estrogen receptor (NICD-ER) fusion from the human ROSA26 locus (Ditadi et al., 2015). These cells ubiquitously express the NICD-ER protein, which can be activated through addition of 4-hydroxytamoxifen (4OHT) to allow translocation to the nucleus and activation of Notch target genes.

Given our hypothesis that Notch signaling impacts cardiomyocyte differentiation during the specification or commitment of early mesoderm, we first determined the effect of Notch induction during early hPSC cardiomyocyte differentiation. 4OHT was added for 24-hour pulses between day 3 and day 6 of differentiation and cardiomyocyte differentiation efficiency was assessed at day 30 . Control cultures yielded $37 \pm 16 \%$ cardiomyocytes at day 30 , as measured by the expression of SIRPA and absence of CD90 (Fig. 3b, left panel) (Dubois et al., 2011). Induction of Notch signaling from days 3-4, 4-5, or 5-6 increased the efficiency of differentiation, with $71 \pm 3.6 \%$ cardiomyocytes being generated after Notch induction from day 5-6 (Fig. $3 \mathrm{~b}$, right panel, quantified in c). Consistent with the data obtained from the in vivo CMes RNA-seq experiments, the strongest effect was seen upon Notch induction at day 5 of differentiation when CMes corresponding to E7.25 mesoderm is present in vitro. We confirmed the increase in cardiomyocyte generation using flow cytometry for the 
bona fide cardiomyocyte marker cardiac troponin T (cTNT) (Fig. 3d, quantified in e). Furthermore, we showed that the differentiation kinetics are not significantly affected upon Notch induction, as the onset of cTNT expression occurs at the same time in control and 4OHT-treated cultures (Fig. 3f).

To determine if Notch is required for cardiomyocyte differentiation under these conditions, we also differentiated cells in the presence of the gamma-secretase inhibitor DAPT, which blocks cleavage of the Notch receptor, thereby inhibiting endogenous signaling activity (Sup. Fig. 4a) (Dovey et al., 2001; Morohashi et al., 2006). Treatment with DAPT for the same $24 \mathrm{~h}$ time periods had no effect on cardiomyocyte differentiation overall (Sup. Fig. 4b, c). To ensure that this observation was not due to the limited time of inhibition or leakiness of the NICD-ER construct, we performed additional inhibition experiments using a human induced PSC (hiPSC) line. We differentiated hiPSCs in the presence of DAPT from days 3-6, covering the key early mesoderm formation and specification events (Sup. Fig. 4d). These experiments confirmed that inhibition of Notch signaling has no effect on cardiomyocyte differentiation efficiency overall (Sup. Fig. 4e, f), and is consistent with recent reports showing no effect from DAPT treatment in standard differentiation conditions (Biermann et al., 2019). This suggests that either Notch signaling is not required for cardiomyocyte differentiation, or that the culture conditions provide a signaling environment that is sufficient to generate cardiomyocytes in the absence of active Notch signaling.

\section{Cardiomyocytes derived from Notch-induced} cardiac mesoderm are developmentally immature

Key signaling events during early differentiation have been shown to impact cardiomyocyte subtype specification, both in vivo and in vitro (Devalla et al., 2015; Hochgreb, 2003; Lee et al., 2017; Xavier-Neto et al., 1999). As we identified the Notch pathway as being enriched in ventricular cardiac mesoderm, we hypothesized that activating Notch would increase ventricular cardiomyocyte specification. To assess how Notch activation in the CMes affects the phenotype of the differentiated cardiomyocytes, we FACS-purified cardiomyocytes at day 30 and performed RNA sequencing. Cardiomyocytes from Notch-induced CMes were distinct from those derived from both untreated (control) and DAPT-treated CMes cultures (Fig. 4a). PCA illustrates that PC1 captures the majority of the variance $(67 \%)$ and largely separates cardiomyocytes from Notch-induced CMes from the other samples, while PC2 (18\% variance captured) reflects experiment-to-experiment variability, even after batch correction with adjustment for hidden covariance. Differential gene expression confirmed the similarity between control and DAPT-treated cultures, yielding no differentially expressed transcripts (FDR $<10 \%$, fold change $>1.5$, base mean $>2$ ). However, cardiomyocytes from Notch-induced cultures express 1,554 transcripts differentially (443 upregulated, green points; 1,111 downregulated, gray points; FDR $<10 \%$, fold change $>1.5$ ) when compared to control cardiomyocytes (Fig. 4b).

As Notch was enriched in vCMes in vivo, we analyzed whether Notch induction in hPSC-derived CMes biases differentiation toward chamber-specific fates. Expression of several well-established atrial and ventricular marker genes did not show a consistent trend, suggesting that Notch signaling does not act to induce a chamber-specific lineage specification mechanism at this early stage of development (Fig. 4c, d) (Cyganek et al., 2018). Interestingly, the expression of MYL2 (ML$\mathrm{C} 2 \mathrm{v}$ ), a ventricular-specific gene expressed during 

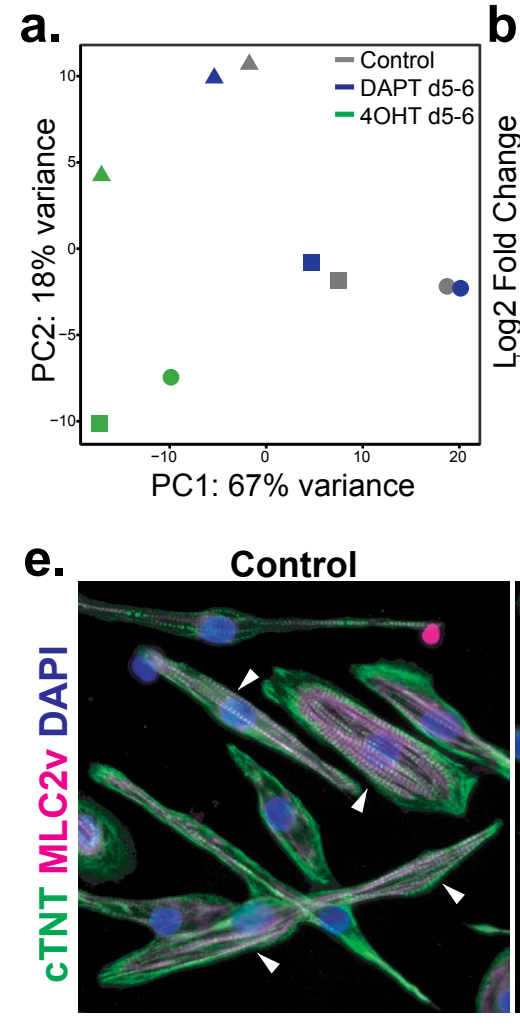

\section{C.}

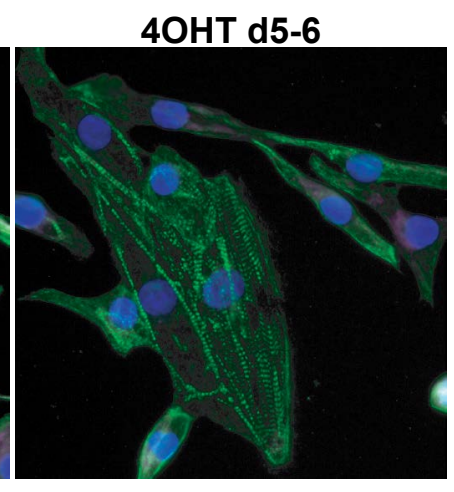

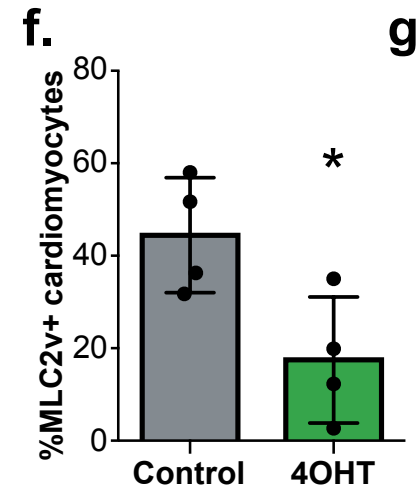

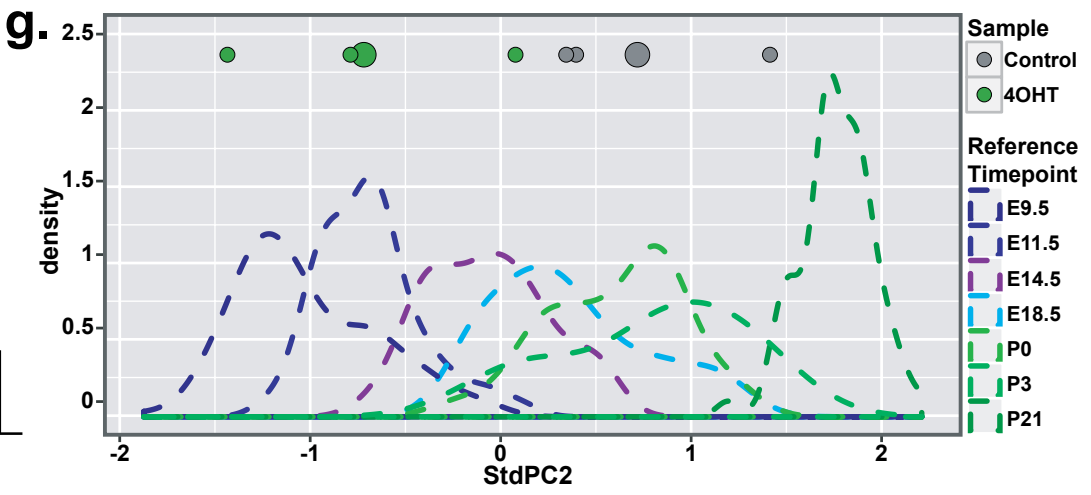

Figure 4: Gene expression analysis of cardiomyocytes derived from control or Notch-perturbed cardiac mesoderm. (a) Principal component analysis of RNA-sequencing data of cardiomyocytes from control, Notch inhibited (DAPT d5-6), or Notch induced (4OHT d5-6) cardiac mesoderm. Colors indicate cell type, symbols indicate replicates. (b) MA plot showing differentially expressed genes between cardiomyocytes from control or Notch induced cultures. (c-d) Gene expression z-scores for representative atrial (c) or ventricular (d) marker genes (genes taken from (Cyganek et al., 2018)). (e) Immunofluorescence analysis of cardiomyocytes from control or Notch induced cultures with antibodies against cTNT and the ventricular marker MLC2v. Arrowheads indicate MLC2v-expressing cardiomyocytes. (f) Flow cytometric quantitation of MLC2v-expressing cardiomyocytes from control or Notch induced cultures. (g) PCA-based determination of cardiomyocyte maturation. Reference stage data (dotted lines) is adapted from (DeLaughter et al., 2016). PC values of comparison data for individual replicates are shown as small circles and averages shown as large circles.

late differentiation, is downregulated in cardiomyocytes from Notch-induced CMes cultures compared to control (Fig. 4c). We confirmed reduced MLC2 $v$ expression at the protein level through immunofluorescence analyses and quantification by flow cytometry (Fig. 4e, f). However, no difference was seen in IRX4 expression, the earliest expressed ventricular-specific transcription factor, suggesting that while ventricular differentiation is compromised, fate acquisition may be unchanged (Fig. 4c).

As an alternative hypothesis, we explored whether the early induction of Notch signaling changes the maturation state of the resulting cardiomyocytes. MYH6 and MYH7, which are often used as atrial and ventricular markers, respective- 
ly, are expressed dynamically during development. Specifically, MYH6 is expressed throughout the early heart tube and downregulated as the ventricles develop, concomitant with MYH7 upregulation (England and Loughna, 2013). Supporting our hypothesis, cardiomyocytes from Notch-induced CMes cultures expressed higher levels of $\mathrm{MYH6}$ and lower levels of $M Y H 7$, suggesting that they may be arrested in an immature state when compared to control cardiomyocytes (Fig. 4c, d). To analyze this in an unbiased fashion, we adapted a recently published principal component-based approach to assess the developmental stage of in vitro-derived cardiomyocytes (DeLaughter et al., 2016). This analysis leverages single cell RNAseq data from mouse hearts collected from E9.5 to postnatal day $(P) 21$. By using a selection of genes expressed in a majority of P0 left ventricle cells, principal component analysis separates the data based on developmental time (Fig. 4g, dotted lines). We applied this same analysis to the gene expression data of cardiomyocytes from control and Notch-induced CMes cultures and overlaid the results onto the mouse scRNA-seq reference data to determine their relative developmental maturity. This analysis revealed that the cardiomyocytes from Notch-induced CMes cultures are more similar to cardiomyocytes from earlier developmental stages when compared to control cardiomyocytes (Fig. $4 \mathrm{~g}$ ), suggesting that Notch induction during early differentiation increases cardiomyocyte generation but leads to permanent changes that arrest the cells in an immature state. This finding is in conflict with recent data showing that treatment with polyinosinic-polycytidylic acid (pIC) resulted in an increase in Notch signaling and concomitant maturation of cardiomyocytes (Biermann et al., 2019). However, pIC treatment also inhibited TGF $\beta$ signaling in these experiments, so it is unclear whether Notch has differing effects depend- ing on the broader signaling environment.

Notch acts in a mesoderm-specific manner to enhance cardiomyocyte differentiation

We next investigated the mechanism by which active Notch signaling increases cardiomyocyte differentiation efficiency. Notch is a well-known regulator of proliferation, leading us to hypothesize that the observed increase in cardiomyocytes is due to an expansion of early cardiac precursor cells (Artavanis-Tsakonas et al., 1999). We therefore assessed proliferation over the course of differentiation and compared control and Notch-induced differentiation cultures (Sup. Fig. 5a). Cells were collected daily from day 5-10 and on days 15 and 30 , after being cultured in the presence of the thymidine analog EdU for 24 hours prior to collection and analysis. Cells treated with $4 \mathrm{OHT}$ from days 5-6 showed a small increase in EdU incorporation at day 6 and 7 , but this increase diminished quickly thereafter (Sup. Fig. 5b). We therefore conclude that proliferation is not the key mechanism by which enhanced Notch signaling increases cardiomyocyte differentiation efficiency.

An alternative hypothesis is that Notch induction causes conversion of non-cardiac progenitors to the cardiac lineage. Since little is known about the role of Notch signaling within gastrulation-stage populations, we sought to use the hPSC differentiation system to dissect the mechanism of its action on these cells. Mesoderm can be detected as early as day 4 of differentiation using the cell surface marker CD13, and CXCR4 is used to exclude endoderm from the analysis (Skelton et al., 2016). We first asked whether Notch increases the commitment of mesoderm to the cardiomyocyte fate or whether it can also convert non-mesodermal cells to the cardiac lineage. We FACS purified mesoderm (CD13+CXCR4-) and non-mesoderm (CD13-) populations at day 4 and plated them as 
bioRxiv preprint doi: https://doi.org/10.1101/2020.02.20.958348; this version posted February 20, 2020. The copyright holder for this preprint (which was not certified by peer review) is the author/funder, who has granted bioRxiv a license to display the preprint in perpetuity. It is made available under aCC-BY-NC-ND 4.0 International license.
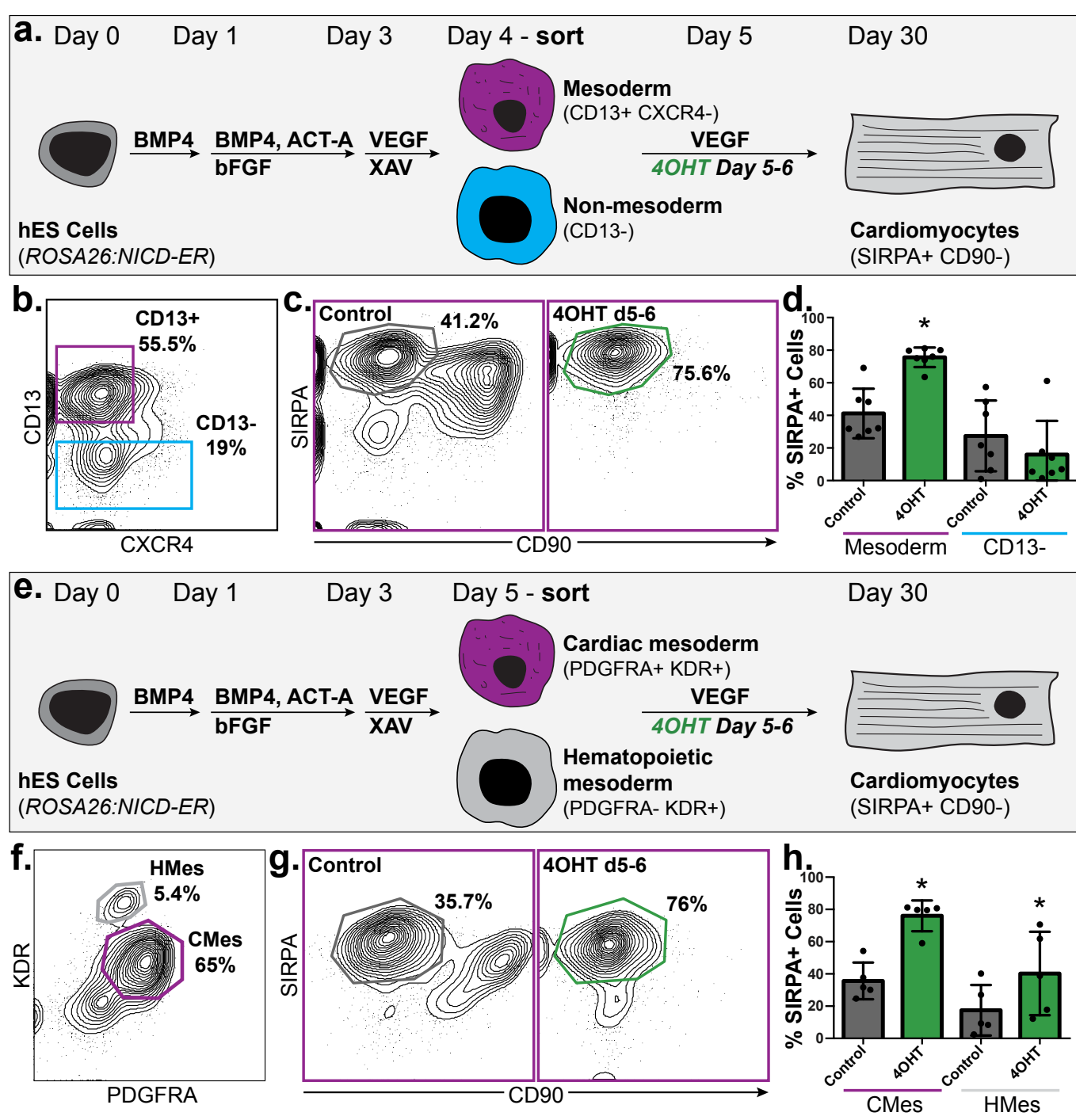

Figure 5: Notch acts in a mesoderm-specific manner to enhance cardiomyocyte differentiation. (a) Schematic of $R O-$ SA26:NICD-ER hPSC-derived day 4 mesoderm purification and cardiomyocyte differentiation. (b) FACS isolation to purify CD13+CXCR4- mesoderm and CD13- non-mesoderm on day 4 of differentiation. (c) Flow cytometric analysis of cardiomyocyte generation from CD13+CXCR4- mesoderm in the absence or presence of Notch induction from day 5-6. (d) Quantification of cardiomyocyte differentiation from mesoderm and non-mesoderm. (e) Schematic of ROSA26:NICD-ER hPSC-derived day 5 mesoderm purification and cardiomyocyte differentiation. (f) FACS isolation to purify PDGFRA+KDR+ cardiac mesoderm and PDGFRA-KDR+ hematopoietic mesoderm. (g) Flow cytometric analysis of cardiomyocyte generation from PDGFRA+K-

$\mathrm{DR}+$ cardiac mesoderm in the absence or presence of Notch induction from day 5-6. (h) Quantification of cardiomyocyte differentiation efficiency from cardiac mesoderm and hematopoietic mesoderm. individual monolayers for continued differentiation. At day 5 , a subset was treated with $4 \mathrm{OHT}$ to induce Notch signaling from day 5-6, as done previously. At day 30 the cultures were analyzed via flow cytometry to quantify differentiation efficiency (Fig. 5a). Differentiation cultures at day 4 were composed of $60 \pm 4.7 \%$ mesoderm and $20 \pm 5 \%$ non-mesodermal cells (Fig. 5b). Mesoderm differentiated in the absence of Notch induction yielded $30 \pm 2.5 \%$ cardiomyocytes at day 30 (Fig. 5c, left). However, when Notch was induced from day 5-6, the efficiency of differentiation was increased to $80 \pm 0.7 \%$ (Fig. 5c, right). As expected, non-mesoderm cells yielded very few cardiomyocytes at day 30. Induction of Notch signaling did not result in an increase when compared to control cultures, indi- cating that non-mesoderm cells are not converted to the cardiac lineage upon Notch activation (Fig. 5d).

To confirm the mesoderm-specific effect seen in these experiments, we differentiated hP. SCs into definitive endoderm progenitors, induced Notch, and analyzed for the presence of cardiomyocytes to assess fate conversion (Sup. Fig. 6a). Definitive endoderm was generated through the addition of high amounts of Activin A at days 1 and 4 of differentiation, and cells were treated with $4 \mathrm{OHT}$ from day 5-6, as in previous experiments. This approach yields $49.5 \pm 23.6 \%$ definitive endoderm cells at day 5 of differentiation, as indicated by the expression of CXCR4 and cKIT (Sup. Fig. 6b) (D'Amour et al., 2005; Gouon-Evans et 
al., 2006). A very small number of cardiomyocytes (5.1 $\pm 2 \%$ SIRPA + CD90-) were observed at day 30 , and Notch induction was not effective at specifying additional cardiomyocytes (Sup. Fig. 6c, d). Thus, consistent with the above results, Notch pathway activity has no cardiogenic effect in non-mesoderm cell types, and the increase in cardiomyocyte differentiation observed is due to a mesoderm-specific mechanism.

Our data thus far show that Notch acts in a mesoderm-specific fashion to increase cardiomyocyte differentiation efficiency. We next determined if the effects of Notch signaling are restricted to the cardiac lineage or can convert other mesoderm lineages to the cardiac fate. Other mesoderm progenitors are generated during differentiation of $\mathrm{hP}$ SCs to cardiomyocytes and can be identified by specific cell surface markers. At day 5 of differentiation, cardiac mesoderm can be identified via surface expression of PDGFRA and KDR, consistent with data described the mouse system (Kataoka et al., 1997; Kattman et al., 2006; Kattman et al., 2011; Takakura et al., 1997). Hematopoietic mesoderm is also generated and characterized by high expression of KDR (KDR ${ }^{\text {hi}}$ ). It has been previously shown that mouse PSC-derived hematopoietic mesoderm can be differentiated to cardiomyocytes through Notch induction (Chen et al., 2008). We therefore wanted to determine if Notch signaling is sufficient to induce cardiomyocyte differentiation from hematopoietic mesoderm in human cells as well. We FACS purified CMes (PDGFRA+KDR+) and HMes $\left(K^{2}{ }^{\text {hi }}\right)$ at day 5 and plated each as monolayers for continued differentiation. A subset was treated with $4 \mathrm{OHT}$ to induce Notch signaling at the time of plating, and cardiomyocyte generation was measured at day 30 (Fig. 5e). Day 5 cultures comprised $53.8 \pm 17 \%$ CMes and $4.7 \pm 1.1 \%$ HMes (Fig. 5f). CMes that was differentiated without Notch induction gave rise to $35.7 \pm 11.4 \%$ SIR-
PA+CD90- cardiomyocytes, similar to the control CD13+CDCR4- day 4 mesoderm (Fig. $5 \mathrm{~g}$, left). Again, Notch induction increased this efficiency to $76 \pm 9.5 \%$ (Fig. $5 \mathrm{~g}$, right). We also observed a statistically significant increase when differentiating HMes into cardiomyocytes in the presence of Notch signaling as compared to control, consistent with previous data from the mouse PSC system (Fig. 5h). Overall, our data from these experiments suggest that germ layer fate switching is not a major contributing factor to the observed increase in differentiation efficiency in Notch induced cultures when compared to controls. Rather, these data support the hypothesis that Notch acts to enhance commitment of the mesoderm progenitors to the cardiac lineage.

The segregation of atrial and ventricular lineages occurs very early during cardiomyocyte differentiation (Bardot et al., 2017b; Devalla et al., 2015; Devine et al., 2014; Lee et al., 2017; Lescroart et al., 2014). All of the experiments to this point have been performed with a protocol that yields primarily ventricular cardiomyocytes. To determine if Notch signaling has similar effects on atrial and ventricular cardiac mesoderm, we next assessed the effect of Notch signaling on atrial cardiomyocyte differentiation. Atrial cardiomyocytes can be differentiated through the addition of all-trans retinoic acid (atRA) at day 3 of differentiation (Sup. Fig. 6e) (Devalla et al., 2015; Lee et al., 2017). In addition to generating atrial-like cardiomyocytes, adding atRA also greatly increases the efficiency of differentiation (Sup. Fig. 6f, g). We did not observe a further increase in cardiomyocyte differentiation efficiency upon Notch induction from day 5-6 (Sup. Fig. 6f, g).

Notch induction is required for cardiomyocyte differentiation in the absence of WNT inhibition Given that Notch signaling appears to act 


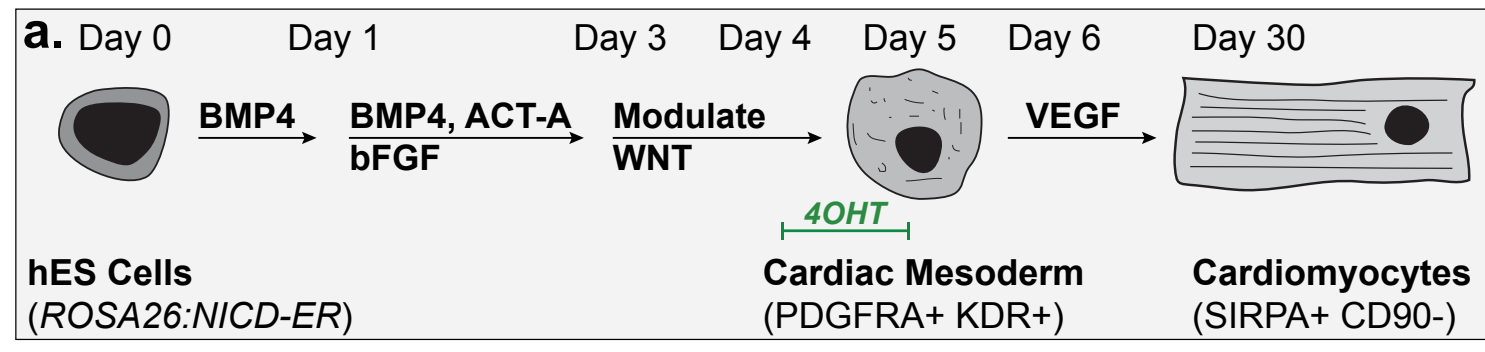

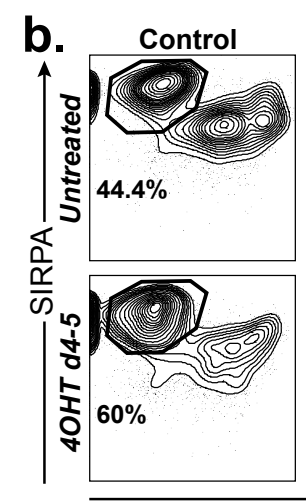

d.
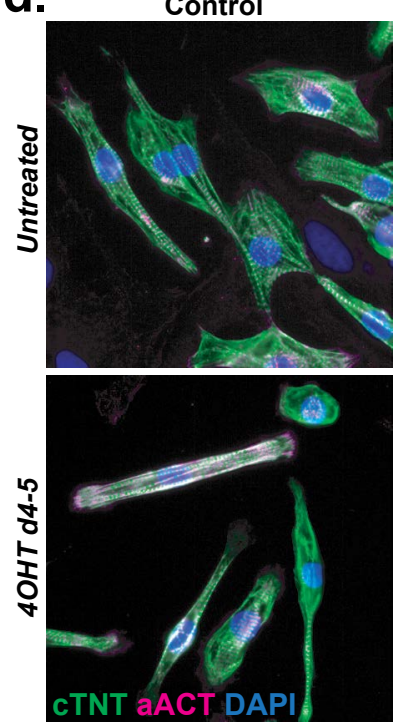
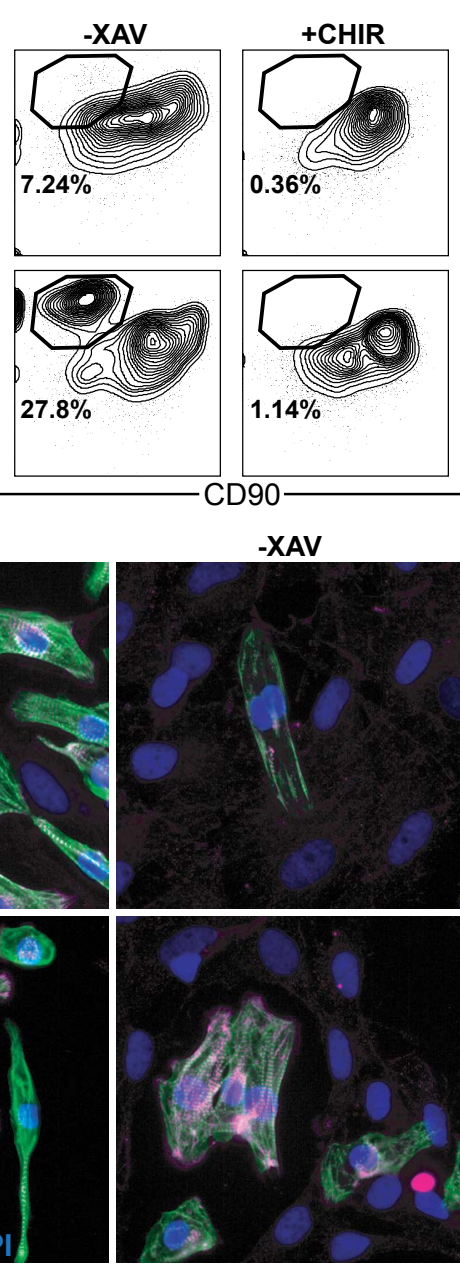
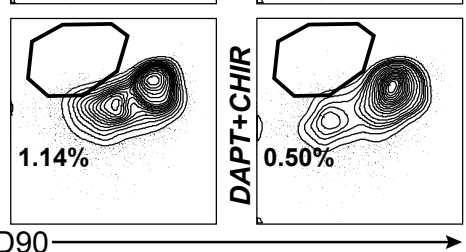

+ CHIR
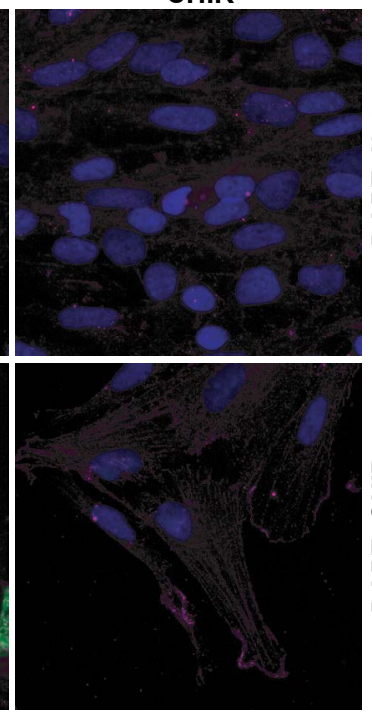

C.

${ }^{80} \%$ Cardiomyocytes at day 30

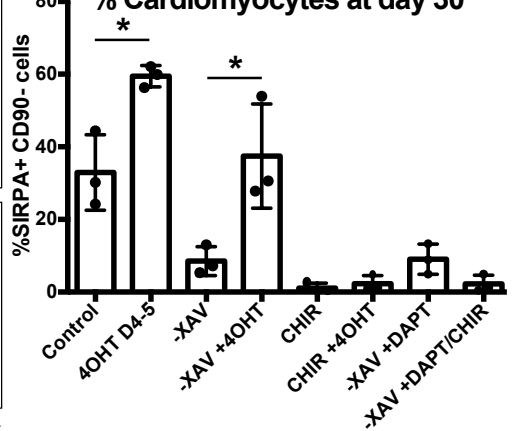

-XAV

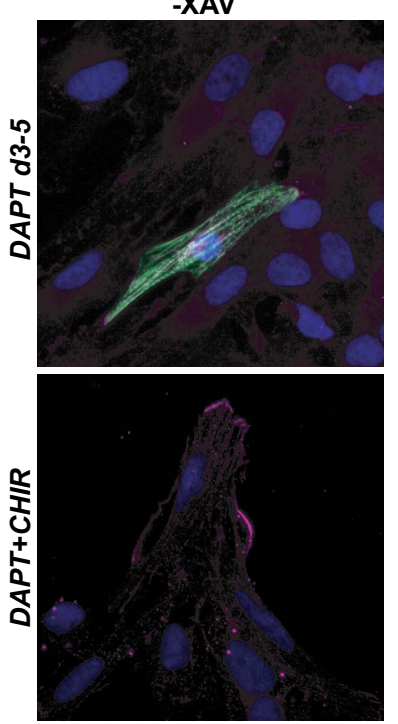

Figure 6: Notch induction is required for cardiomyocyte differentiation in the absence of WNT inhibition. (a) Schematic of ROSA26:NICD-ER hPSC-cardiomyocyte differentiation with WNT and Notch modulation. (b) Flow cytometric analysis of cardiomyocyte generation in the absence of WNT inhibition (-XAV) or presence of WNT induction (CHIR), with and without Notch induction (4OHT) or inhibition (DAPT). (c) Quantification of data in (b). (d) Immunofluorescence analysis on day 30 cardiomyocytes with antibodies against cTNT and aACT.

on mesoderm specifically, we sought to determine whether Notch interacts with other signaling pathways during cardiac commitment. At the time points when Notch-induction was most effective (day 4-5 or 5-6), cells are cultured in the presence of VEGF and the WNT inhibitor XAV939 (days 3 to 5), or VEGF alone (days 5 to 7 ), suggesting that VEGF or WNT signaling may play parallel or additive roles with Notch at this time. It has been shown in vivo that there is a switch from canonical to non-canonical Wnt signaling at this stage, and that Notch may play a role in this process (Guo et al., 2019; Klaus et al., 2012; Miazga and McLaughlin, 2009; Wang et al., 2018). To test whether Notch signaling is sufficient to specify mesoderm to the cardiac lineage, we first excluded VEGF during differentiation, and added $4 \mathrm{OHT}$ to induce Notch signaling from day 4-5 (Sup. Fig. 7a). Culturing cells in the absence of VEGF throughout differentiation had no effect on overall cardiomyocyte 
generation efficiency, whether or not Notch was induced (Sup. Fig. 7b, c). Immunofluorescence analysis for cTNT and alpha-actinin (aACT) confirmed that phenotypically normal cardiomyocytes were present in the absence of VEGF (Sup. Fig. $7 d$ ), leading us to conclude that under these culture conditions VEGF has no effect on hPSC differentiation to cardiomyocytes.

However, cells differentiated without XAV939 yielded less than $8.5 \pm 4 \%$ cardiomyocytes at day 30 , confirming that WNT inhibition is essential for commitment of cells to the cardiac lineage, as shown previously (Fig. 6a, b) (Lian et al., 2013). Addition of DAPT failed to enhance this effect (Fig. 6b). Interestingly, induction of Notch from day 4-5 in the absence of XAV939 was able to restore cardiomyocyte differentiation to control levels (Fig. $6 b)$. This suggests that Notch is required for cardiomyocyte differentiation in the absence of WNT inhibition, and that Notch may be acting to inhibit WNT signaling during that process. To further examine that hypothesis, we cultured cells in the presence of the GSK3B inhibitor CHIR, which stabilizes $\mathrm{B}$-catenin and results in constitutive activation of canonical WNT signaling. Addition of $\mathrm{CHIR}$ completely blocked cardiomyocyte differentiation, and Notch induction failed to rescue this effect (Fig. 6b). Quantification of these data confirm the interaction of Notch and WNT signaling in these experiments (Fig. 6c). In all conditions where cardiomyocytes were detected by flow cytometry analysis, immunofluorescence analysis for cTNT and AACT confirmed the presence of phenotypically normal cardiomyocytes (Fig. 6d). Overall, our data support a model in which Notch signaling acts to increase cardiac lineage commitment by inhibiting WNT activity and show that Notch signaling on its own is sufficient to commit mesoderm to the cardiac lineage.

\section{Discussion}

We have previously identified a ventricular-specific cardiac progenitor population specified during gastrulation through the use of Foxa2 lineage-tracing (Bardot et al., 2017b). Here, we have expanded on these findings and studied the heterogeneity of cardiac mesoderm cell populations and the early steps of cardiac mesoderm differentiation both in vivo and in vitro. Our study focused on a developmental stage occurring at E7.25 in vivo or day 5 of hPSC differentiation, which is likely prior to lineage commitment. This stage encompasses the earliest steps of cardiac specification and captures the differential embryonic signaling environment cells are exposed to as they migrate anteriorly to form the cardiac crescent. Foxa2 lineage-tracing has enabled us to distinguish, at this early stage, the mesoderm populations that will give rise to ventricular cells (vCMes) versus atrial cells (aCMes) later during development, and therefore gives us the ability to investigate early versus late differentiating cells of the cardiovascular lineage.

The in vivo RNA-seq analysis we performed suggest that cardiac mesoderm from the mouse embryo and from mESCs are overall similar after accounting for technical differences. Notch signaling appears to be active in all cardiac mesoderm populations, though cells further along the differentiation trajectory in both systems (Foxa2-hCD4+ CMes in vitro, Foxa2Cre:YFP+ CMes in vivo) express higher levels of some Notch pathway components. Other studies have shown that Notch1 is expressed in early Mesp1+ cardiac precursors that are fated for the endothelial lineage, but did not address whether Notch signaling plays a role in that lineage decision (Lescroart et al., 2018). We observed expression of Notch1-3 in both aCMes and vCMes, though we are unable to determine the relationship between receptor expression and lin- 
eage decision from our data. By taking advantage of the hPSC cardiomyocyte differentiation model, we were able to show that inducing Notch signaling in hPSC-derived cardiac mesoderm enhances cardiomyocyte differentiation overall. Such transient Notch induction appears not to affect atrial vs. ventricular fate decisions, but rather increases cardiomyocyte generation efficiency more generally. This suggests that while Notch components are enriched in ventricular-fated cardiac mesoderm, we likely capture a snapshot of development when Notch signaling is being consecutively activated in migratory mesoderm. While all cardiac mesoderm will be subjected to Notch signaling, the early differentiating ventricular progenitors receive the signal first, while later-specified atrial progenitors are exposed to it subsequently.

Within the heart, Notch is known to act at numerous stages during development in different organisms (High and Epstein, 2008). Aberrant activation of Notch signaling in cardiac precursors causes an enlargement of the left ventricle and may negatively impact maturation of the forming cardiomyocytes (Watanabe, 2006). While Notch activation in the cardiac lineage showed a hyperplasic phenotype, induction of Notch signaling in the epiblast has a negative overall impact on mesoderm specification and organization (Souilhol et al., 2015). Data from Xenopus suggests that active Notch signaling is important during a brief time window between gastrulation and differentiation, and that this timing acts to coordinate progenitor maintenance and differentiation. Specifically, premature dampening of the Notch pathway results in cardiac progenitors upregulating the differentiation program prior to reaching the midline of the embryo (Miazga and McLaughlin, 2009). This is consistent with our data showing that transient Notch induction specifically at the cardiac mesoderm stage enhances cardiomyocyte differentiation ef- ficiency. We also showed that the inductive effects of Notch signaling are specific to mesoderm, and that Notch was insufficient to convert endoderm to the cardiac lineage.

Knowing that the differentiation protocol used provides a highly cardiogenic signaling environment, we sought to determine the interaction between Notch and other relevant pathways. Despite the expression of VEGFR2 (KDR) in both in vivo and in vitro mesoderm, removing VEGF had little effect on hPSC differentiation overall. This is consistent with data from the mouse embryo showing that while $K d r$ is required for the formation of hematopoietic lineages, it is not required for cardiac progenitor formation (Ema et al., 2006). Notch is known to interact with other signaling pathways, particularly the BMP and Wnt pathways. Notch has been shown to activate the non-canonical Wnt5a ligand later during heart development, further suggesting a link between these two pathways (Wang et al., 2018). It was previously shown that mouse ESC-derived $\mathrm{Kdr}+$ cells fated to the hematopoietic and vascular lineages can be redirected to the cardiac lineage via Notch activation (Chen et al., 2008). Gene expression profiling revealed that this was in part due to upregulation of BMP pathway genes and downregulation of canonical Wnt signaling, ultimately mimicking the signaling cocktail used to generate cardiomyocytes from PSCs. In the embryo, the overlying endoderm provides high levels of Wnt-inhibitory proteins to the developing mesoderm, and proper endoderm development has been shown to be critical for heart development (Marvin et al., 2001; Nascone and Mercola, 1995; Schneider and Mercola, 2001; Schultheiss et al., 1995).

In accordance with an important role for Wnt inhibition during cardiac mesoderm specification, our analysis reveals expression of secreted Wnt inhibitory proteins and non-canonical ligands, 
with Sfrp1 being enriched in early-migrating ventricular cardiac mesoderm (Figure 2b). Consistent with the importance of that interaction, we found that failing to inhibit WNT in vitro almost completely eliminated cardiomyocyte differentiation. Interestingly, Notch induction is sufficient to rescue hPSC differentiation to control levels, providing evidence that these two pathways interact during cardiac mesoderm commitment. We therefore believe that Notch in the cardiac mesoderm is acting in concert with the overlying endoderm to establish a Wnt-inhibitory zone as cells approach the anterior side of the embryo, and that inducing Notch during hPSC differentiation recapitulates aspects of this mechanism. These findings have implications for therapeutic uses, as a fuller understanding of the in vivo signaling environment may lead to development of cardiomyocyte differentiation protocols with improved efficiency and cardiomyocyte subtype selectivity.

Our experiments illustrating enhanced cardiomyocyte differentiation after Notch induction nicely corroborate our gene expression profiling in vivo and suggest that Notch plays a role during early mammalian heart development, a stage that is technically challenging to manipulate in a temporally and spatially controlled manner. Going forward, it will be important to dissect the detailed mechanism for how this occurs, and whether Notch signaling in the cardiac mesoderm is broadly cardiogenic. While studies have looked at the effects of Notch signaling on mouse development, these have largely been done using constitutive Notch activation (Souilhol et al., 2015; Watanabe, 2006). It would be of great interest to perturb Notch signaling within the early cardiac lineage in a temporally controlled and reversible manner, as has been done in other model organisms (Miazga and McLaughlin, 2009).

In summary, we have used a combination of mouse embryo and human PSC approaches to dissect the early steps of heart progenitor specification. By performing whole transcriptome analysis on in vivo cardiac mesoderm, we identified an enrichment for Notch pathway components in the early-differentiating ventricular lineage. Using human pluripotent stem cell directed differentiations, we found that transient Notch induction at the cardiac mesoderm stage increases the efficiency of cardiomyocyte differentiation. Surprisingly, Notch activation does not impact the proportion of ventricular cardiomyocytes formed, but rather yields cells less developmentally mature than control counterparts. Finally, we show that the cardiac-inducing effect of Notch signaling is restricted to mesodermal cells and may act to inhibit Wnt signaling. These data reveal a role for Notch signaling in committing early mesoderm to the cardiac lineage in both mouse and human systems.

\section{Materials and methods}

\section{Mice}

The Foxa2Cre mouse line (C57BL/6J) was generated and shared with us by Dr. Heiko Lickert (Horn et al., 2012). Rosa26-YFP (006148; C57BL/6J), Rosa26-mTmG (007676; C57BL/6J), and Rosa26-H2B-mCherry (023139; B6/129S) mice were all obtained from The Jackson Laboratory. Studies were performed on mixed backgrounds as dictated by strain availability. For time-course experiments, the day of plug identification corresponds to embryonic day 0.5 (E0.5). All animals were housed in facilities operated by the Center for Comparative Medicine and Surgery (CCMS) at Icahn School of Medicine. All animal experiments were conducted in accordance with the guidelines and approval of the Institutional Animal Care and Use Committee at Icahn School of Medicine at Mount Sinai. 


\section{Cell lines}

The ROSA26:NICD-ER hESC line was generated and generously shared with us by Dr. Paul Gadue (Ditadi et al., 2015; Gadue et al., 2006). The MYL2-mEGFP WT hiPSC line was obtained from the Coriell Institute (Line no. AICS-0060-027).

\section{Histology and Immunofluorescence}

Embryos were collected from timed pregnancies and either fixed in 4\% PFA, washed with phosphate buffered saline (PBS) and equilibrated in $30 \%$ sucrose (Sigma-Aldrich) before embedding, or embedded fresh into OCT (Electron Microscopy Sciences). Tissues were subsequently cut into $10 \mu \mathrm{m}$ sections using a Leica Cryostat. Slides were fixed for $10 \mathrm{~min}$ in 4\% PFA and incubated for $1 \mathrm{~h}$ in blocking solution (PBS with $0.1 \%$ Triton and $1 \%$ BSA). Primary antibodies were diluted in blocking solution and incubations were carried out for $1 \mathrm{~h}$ at room temperature or overnight at $4^{\circ} \mathrm{C}$, followed by incubation in secondary antibody for $1 \mathrm{~h}$ at room temperature. Slides were then counterstained with DAPI and mounted using glycerol-based $\mathrm{nPG}$ (Sigma-Aldrich, P3130) antifade mounting media.

For whole mount immunofluorescence, embryos were collected, immersion-fixed in 4\% PFA overnight, and washed with PBS. Embryos were blocked for $>4 \mathrm{~h}$ in blocking solution (PBS with $0.1 \%$ Triton and $1 \%$ BSA). Primary antibodies were diluted in blocking solution and incubations were carried out overnight at $4^{\circ} \mathrm{C}$. Embryos were washed three times in PBS-T (PBS with $0.1 \%$ Triton) for $2 \mathrm{~h}$ each, followed by incubation in secondary antibody overnight at $4^{\circ} \mathrm{C}$. Embryos were then counterstained with DAPI and allowed to equilibrate in $\mathrm{nPG}$ antifade mounting media before mounting. Embryos were mounted on slides using double-stick tape and coverslips. The following primary antibodies and dilutions were used:
anti-GFP (abcam, 1:500); anti-RFP (Rockland, 1:500); anti-cTnT (ThermoSci, 1:300); anti-Mlc2v (Proteintech, 1:100). Secondary antibodies conjugated with Alexa dyes were obtained from Jackson Immunoresearch and used at 1:500.

Fluorescence images were obtained using either Leica DM6000 or DM5500B microscopes. Confocal microscopy was performed using a Zeiss 780 microscope. Images were processed using Imaris 8, FIJI ImageJ, or Adobe Photoshop software (Bardot et al., 2017a).

\section{Human pluripotent stem cell differentiations}

Mesoderm/Cardiac: Embryoid bodies (EBs) were generated from ESC lines using collagenase $B$ (Roche) and cultured in RPMI 1640 base media (ThermoFisher) containing 0.5X B27 supplement (ThermoFisher), 2mM glutamine (Gibco-BRL), $1 \mathrm{mM}$ ascorbic acid (Sigma), and 4x10-4 M monothioglycerol (Sigma)) with BMP4 $(1 \mathrm{ng} / \mathrm{ml}$, R\&Dsystem) and Thiazovivin ( $2 \mu \mathrm{M}$, Millipore). On day 1 , EBs were harvested and resuspended in induction medium (base media with basic fibroblast growth factor (bFGF; $2.5 \mathrm{ng} / \mathrm{ml}$, R\&Dsystem), activin A (20 ng/ml, R\&Dsystem) and BMP4 (20 ng/ $\mathrm{ml})$ ). On day 3, the EBs were harvested and resuspended in base media with vascular endothelial growth factor (VEGF; $10 \mathrm{ng} / \mathrm{ml}$, R\&Dsystem) and XAV939 (10 $\mu \mathrm{M}$, Stemolecule). On day 3, the EBs were harvested and resuspended in base media with vascular endothelial growth factor (VEGF; 10 $\mathrm{ng} / \mathrm{ml}, \mathrm{R} \&$ Dsystem). On day 8 and thereafter, EBs were cultured in base media until day 30 for further analysis.

Endoderm: Human pluripotent stem cells were differentiated into the endoderm lineage as previously described (Goldman et al., 2013). Briefly, cells were dissociated using collagenase $B$ (Roche) and 
cultured in low cluster plates to allow EB formation in in RPMI 1640 base media (ThermoFisher) supplemented with BMP4 (3 ng/ml, R\&D Systems) and Thiazovivin ( $2 \mu \mathrm{M}$, Millipore). At day 1 the medium was changed to base media supplemented with Activin A (100 ng/ml, R\&D Systems), basic FGF (bFGF, $2.5 \mathrm{ng} / \mathrm{ml}$, R\&D Systems), and BMP4 $(0.5 \mathrm{ng} / \mathrm{ml}, \mathrm{R} \& D$ Systems $)$. At day 4 , the medium was changed to the base media supplemented with Activin A (100 ng/ml), bFGF (2.5 ng/ml), and vascular endothelial growth factor (VEGF, $10 \mathrm{ng} /$ $\mathrm{ml}, \mathrm{R} \& \mathrm{D}$ Systems). On day 5 EBs were dissociated and analyzed for CXCR4+ cKIT+ cells. On day 6 and thereafter, EBs were cultured in base media until day 30 for further analysis.

Isolation and differentiation of early subpopulations during differentiation

On day 4 of cardiac differentiation, EBs were dissociated using TrypLE (Invitrogen). CXCR4-CD13+ and CXCR4-CD13+ cells were sorted and cultured in matrigel-coated 96 well plates at $1 \times 105$ cells per well in base media with vascular endothelial growth factor (VEGF; $10 \mathrm{ng} / \mathrm{ml}$ ), XAV939 (10microM), and Thiazovivin $(2 \mu \mathrm{M})$. On day 5 , the media was changed to base media with vascular endothelial growth factor (VEGF; $10 \mathrm{ng} / \mathrm{ml}$ ), and cultures were maintained in this media until day 8 of differentiation, when cells were cultured in base media only, with media changes every 3 days until day 30 , when cells were analyzed.

On day 5 of cardiac differentiation, EBs were dissociated using TrypLE (Invitrogen). PDGFRA+KDR+, PDGFRA-KDR+ and PDGFRA-KDR- cells were sorted and cultured in matrigel-coated 96 well plates at $1 \times 105$ cells per well in base media with vascular endothelial growth factor (VEGF; $10 \mathrm{ng} / \mathrm{ml})$ and Thiazovivin $(2 \mu \mathrm{M})$. On day 6 , the media was changed to base media with vascular endothelial growth factor (VEGF; $10 \mathrm{ng} / \mathrm{ml}$ ), and cultures were maintained in this media until day 8 of differentiation, when cells were cultured in base media only, with media changes every 3 days until day 30 , when cells were analyzed.

\section{Flow cytometry analysis and cell sorting}

Mouse embryos: E7.25 embryos were dissociated with TrypLE. Cells were washed in staining solution (DMEM with $0.1 \% \mathrm{BSA}$ ), pelleted, and resuspended in staining solution. Antibodies were diluted in staining solution and cells were incubated on ice for $30 \mathrm{~min}$. Cells were then washed, filtered, and resuspended in staining solution for analysis or cell sorting.

Day 3 to day 15 hPSC-EBs: EBs generated from hPSC differentiation experiments were dissociated with $0.25 \%$ trypsin/EDTA.

Day 20 and older hPSC-EBs: EBs were incubated in collagenase type $\|(1 \mathrm{mg} / \mathrm{ml}$; Worthington) in Hanks solution ( $\mathrm{NaCl}, 136 \mathrm{Mm}$; $\mathrm{NaHCO}, 4.16$ $\mathrm{mM}$; NaPO4, $0.34 \mathrm{mM}$; KCl, $5.36 \mathrm{mM}$; KH2PO4, $0.44 \mathrm{mM}$; dextrose, $5.55 \mathrm{mM}$; HEPES, $5 \mathrm{mM}$ ) overnight at $37^{\circ} \mathrm{C}$. The EBs were pipetted gently to dissociate the cells, washed, and resuspended in staining solution (PBS with $0.1 \% \mathrm{BSA}$ ) and then filtered. Antibodies were diluted in staining solution and cells were incubated on ice for $30 \mathrm{~min}$. Cells were then washed and resuspended in staining solution for analysis or cell sorting.

Cell counts were collected using a LSRII (BD Biosciences) and data was analyzed using the FlowJo software.

The following antibodies and dilutions were used: anti-mouse Pdgfra-BV421 (BD, 1:100); anti-mouse Kdr-PE-Cy7 (BD, 1:100); anti-mouse 
Epcam (eBioscience, 1:100); anti-cardiac Troponin $\mathrm{T}$ (ThermoSci, 1:100); anti-human CD172a/b (SIRPa/ $\beta$ ) (Biolegend; clone SE5A5; 1:200) anti-human CD13 (Biolegend; clone WM15; 1:1,000), anti-human CD90 (Biolegend, clone 5E10; 1:200); anti-human CD140a (PDGFRa) (Biolegend, clone 16A1; 1:250); anti-human CD309 (KDR/VEGFR2) (Biolegend, clone 7D4-6; 1:200); anti-human CD184 (CXCR4) (Biolegend, clone 12G5; 1:500).

\section{cDNA library preparation and RNA-sequencing} Total RNA obtained from FACS-sorted E7.5 mouse embryonic populations was purified with the Absolutely RNA nanoprep kit (Agilent) and quantified as above. Sample cDNA libraries were prepared using the Ovation RNA-Seq System V2 (NuGEN). First strand cDNA was analyzed by qPCR, and only samples with faithful expression of the markers used for FACS isolation were submitted for sequencing.

Total RNA from FACS-sorted hPSC-derived cardiomyocytes was isolated using the Quick-RNA microprep kit (Zymo Research) and submitted to the NYU Genomics Facility for QC, library generation, and sequencing.

\section{RNA-seq data processing}

Quality analysis of raw sequencing reads was performed using FastQC (http://www.bioinformatics. bbsrc.ac.uk/projects/fastqc/). Reads were then aligned to the mouse reference genome (mm10) for mouse samples or the human reference genome (hg19) for human samples using STAR with the two-pass setting (Dobin et al., 2013), and a database of known splice junctions from the ENCODE mm10/GRCm38 annotation for the initial alignment (http://www.gencodegenes.org/mouse releases/2.html). Picard tools (http://broadinstitute.github.io/picard/) was used to index and re- move duplicate reads from the resulting SAM files, generating 16-25 million unique aligned reads per sample. From the resulting alignments, we used HTSeq (Anders et al., 2015) to generate read counts per transcript, and normalized these and performed differential expression analysis using DESeq2 (Love et al., 2014). Gene ontology analysis was performed using Panther GO (http://geneontology.org/page/go-enrichment-analysis). All heatmaps were generated using the $R$ package pheatmap ( $v$ 1.0.8). PCA and MA plots were generated using the $R$ packages ggplot2 $(v 2.0 .0)$ and DESeq2 ( $v$ 1.10.1). The developmental benchmarking was performed using the $R$ package princomp on Log10 FPKM data, as previously described (DeLaughter et al., 2016).

\section{Statistical analysis}

Statistical analysis was performed using Student's t-test and is presented as statistically significant $\left(^{*}\right)$ with a $p$-value cutoff of $p<0.05$. Unless specified, error bars indicate mean \pm standard deviation.

\section{Acknowledgements}

We would like to thank Dr. Paul Gadue for providing the ROSA26-ICN-ER hESC line and the Foxa2- $h C D 4 \mathrm{mESC}$ line. The Foxa2Cre mice were generated and generously shared by Dr. Heiko Lickert. We greatly appreciate Drs. Dan DeLaughter and Christine Seidman for their assistance with the cardiomyocyte maturity analysis. We would like to acknowledge the ISMMS shared resource facilities, in particular the microscopy (Drs. Deena Benson and Nikos Tzavaras), medical illustration (Ni-ka Ford), flow cytometry (Christopher Bare and Venu Pothula), and stem cell (Dr. Sunita D'Souza) core facilities. Dr. Ariana Heguy and the NYU Genome Technology Center provided guidance and expertise on RNA-sequencing sample preparation 
and performed all RNA-sequencing.

\section{Author contributions}

ESB and NCD designed and performed experiments and analyzed the data. BJ and AJS analyzed the RNA-sequencing data. NW performed experiments and analyzed the data. AR and MR developed adapted protocols for generating RNA-sequencing libraries from in vivo samples. ESB and NCD wrote the manuscript with input from all authors.

\section{Competing interests}

The authors have no competing interest to declare.

\section{Funding}

NCD is funded by NIH NHLBI R01 HL134956. ESB is supported by NIH NHLBI F31 HL136216.

\section{Data availability}

The authors declare that all data supporting the findings of this study are available within the article and its supplementary information files, or from the corresponding author upon request. The RNA sequencing raw reads and deSeq2 data have been deposited in the NCBI GEO database under the accession codes GSE143226 and GSE143227.

\section{References}

Anders, S., Pyl, P. T. and Huber, W. (2015). HTSeq-A Python framework to work with high-throughput sequencing data. Bioinformatics 31, 166-169.

Artavanis-Tsakonas, S., Rand, M. D. and Lake, R. J. (1999). Notch signaling: Cell fate control and signal integration in development. Science (80-. ). 284, 770-776.

Bardot, E., Tzavaras, N., Benson, D. L. and Dubois, N. C. (2017a). Quantitative Whole-mount Immunofluorescence Analysis of Cardiac Progenitor Populations in Mouse Embryos. J. Vis. Exp. 1-7.
Bardot, E., Calderon, D., Santoriello, F., Han, S., Cheung, K., Jadhav, B., Burtscher, I., Artap, S., Jain, R., Epstein, J., et al. (2017b). Foxa2 identifies a cardiac progenitor population with ventricular differentiation potential. Nat. Commun. 8,.

Biermann, M., Cai, W., Lang, D., Hermsen, J., Profio, L., Zhou, Y., Czirok, A., Isai, D. G., Napiwocki, B. N., Rodriguez, A. M., et al. (2019). Epigenetic Priming of Human Pluripotent Stem Cell-Derived Cardiac Progenitor Cells Accelerates Cardiomyocyte Maturation. Stem Cells.

Calderon, D., Bardot, E. and Dubois, N. (2016). Probing early heart development to instruct stem cell differentiation strategies. Dev. Dyn. 245, 1130-1144.

Chen, V. C., Stull, R., Joo, D., Cheng, X. and Keller, G. (2008). Notch signaling respecifies the hemangioblast to a cardiac fate. Nat. Biotechnol. 26, 1169-1178.

Cyganek, L., Hasenfuss, G., Guan, K., Cyganek, L., Tiburcy, M., Sekeres, K. and Gerstenberg, K. (2018). Deep phenotyping of human induced pluripotent stem cell - derived atrial and ventricular cardiomyocytes Find the latest version: Deep phenotyping of human induced pluripotent stem cell - derived atrial and ventricular cardiomyocytes. JCl insight 3, e99941.

D'Amour, K. A., Agulnick, A. D., Eliazer, S., Kelly, O. G., Kroon, E. and Baetge, E. E. (2005). Efficient differentiation of human embryonic stem cells to definitive endoderm. Nat. Biotechnol.

DeLaughter, D. M., Bick, A. G., Wakimoto, H., McKean, D., Gorham, J. M., Kathiriya, I. S., Hinson, J. T., Homsy, J., Gray, J., Pu, W., et al. (2016). Single-Cell Resolution of Temporal Gene Expression during Heart Development. Dev. Cell 39, 480-490.

Devalla, H. D., Schwach, V., Ford, J. W., Milnes, J. T., El-Haou, S., Jackson, C., Gkatzis, K., Elliott, D. A., Chuva de Sousa Lopes, S. M., Mummery, C. L., et al. (2015). Atrial-like cardiomyocytes from human pluripotent stem cells are a robust preclinical model for assessing atrial-selective pharma- 
cology. EMBO Mol. Med. 7, 394-410.

Devine, W. P., Wythe, J. D., George, M., Koshiba-Takeuchi, K. and Bruneau, B. G. (2014). Early patterning and specification of cardiac progenitors in gastrulating mesoderm. Elife 3, e03848.

Ditadi, A., Sturgeon, C. M., Tober, J., Awong, G., Kennedy, M., Yzaguirre, A. D., Azzola, L., Ng, E. S., Stanley, E. G., French, D. L., et al. (2015). Human definitive haemogenic endothelium and arterial vascular endothelium represent distinct lineages. Nat. Cell Biol. 17, 580-591.

Dobin, A., Davis, C. A., Schlesinger, F., Drenkow, J., Zaleski, C., Jha, S., Batut, P., Chaisson, M. and Gingeras, T. R. (2013). STAR: Ultrafast universal RNA-seq aligner. Bioinformatics 29, 15-21.

Dovey, H., John, V., Anderson, J., Chen, L., de Saint Andrieu, P., Fang, L., Freedman, S., Folmer, B., Goldbach, E., Holsztynska, E., et al. (2001). Functional gamma-secretase inhibitors reduce beta-amyloid peptide levels in brain. J. Neurochem. 76, 173-181.

Dubois, N. C., Craft, A. M., Sharma, P., Elliott, D. A., Stanley, E. G., Elefanty, A. G., Gramolini, A. and Keller, G. (2011). SIRPA is a specific cell-surface marker for isolating cardiomyocytes derived from human pluripotent stem cells. Nat. Biotechnol. 29, 1011-1018.

Ema, M., Takahashi, S. and Rossant, J. (2006). Deletion of the selection cassette, but not cis-acting elements, in targeted Flk1-lacZ allele reveals Flk1 expression in multipotent mesodermal progenitors. Blood 107, 111-117.

England, J. and Loughna, S. (2013). Heavy and light roles: Myosin in the morphogenesis of the heart. Cell. Mol. Life Sci.

Gadue, P., Huber, T. L., Paddison, P. J. and Keller, G. M. (2006). Wnt and TGF-beta signaling are required for the induction of an in vitro model of primitive streak formation using embryonic stem cells. Proc. Natl. Acad. Sci. 103, 16806-16811.

Garcia-Martinez, V. and Schoenwolf, G. C. (1993).
Primitive-streak origin of the cardiovascular system in avian embryos. Dev. Biol. 159, 706-719.

Gouon-Evans, V., Boussemart, L., Gadue, P., Nierhoff, D., Koehler, C. I., Kubo, A., Shafritz, D. A. and Keller, G. (2006). BMP-4 is required for hepatic specification of mouse embryonic stem cell-derived definitive endoderm. Nat. Biotechnol. 24, 1402-1411.

Guo, Y., Dorn, T., Kühl, S. J., Linnemann, A., Rothe, M., Pfister, A. S., Vainio, S., Laugwitz, K. L., Moretti, A. and Kühl, M. (2019). The Wnt inhibitor Dkk1 is required for maintaining the normal cardiac differentiation program in Xenopus laevis. Dev. Biol. 449, 1-13.

High, F. A. and Epstein, J. A. (2008). The multifaceted role of Notch in cardiac development and disease. Nat. Rev. Genet. 9, 49-61.

Hochgreb, T. (2003). A caudorostral wave of RALDH2 conveys anteroposterior information to the cardiac field. Development 130, 5363-5374.

Horn, S., Kobberup, S., Jorgensen, M. C., Kalisz, M., Klein, T., Kageyama, R., Gegg, M., Lickert, H., Lindner, J., Magnuson, M. A., et al. (2012). Mind bomb 1 is required for pancreatic -cell formation. Proc. Natl. Acad. Sci. 109, 7356-7361.

Inagaki, T., Garcia-Martinez, V. and Schoenwolf, G. C. (1993). Regulative ability of the prospective cardiogenic and vasculogenic areas of the primitive streak during avian gastrulation. Dev. Dyn. 197, 57-68.

Kataoka, H., Takakura, N., Nishikawa, S., Tsuchida, K., Kodama, H., Kunisada, T., Risau, W., Kita, T. and Nishikawa, S. L. (1997). Expressions of PDGF receptor alpha, c-Kit and Flk1 genes clustering in mouse chromosome 5 define distinct subsets of nascent mesodermal cells. Dev. Growth Differ. 39, 729-740.

Kattman, S. J., Huber, T. L. and Keller, G. M. M. (2006). Multipotent Flk-1+ Cardiovascular Progenitor Cells Give Rise to the Cardiomyocyte, Endothelial, and Vascular Smooth Muscle Lineages. Dev. Cell 11, 723-732. 
Kattman, S. J., Witty, A. D., Gagliardi, M., Dubois, N. C., Niapour, M., Hotta, A., Ellis, J. and Keller, G. (2011). Stage-specific optimization of activin/nodal and BMP signaling promotes cardiac differentiation of mouse and human pluripotent stem cell lines. Cell Stem Cell 8, 228-240.

Keegan, B. R. (2004). Organization of cardiac chamber progenitors in the zebrafish blastula. Development 131, 3081-3091.

Klaus, A., Muller, M., Schulz, H., Saga, Y., Martin, J. F. and Birchmeier, W. (2012). Wnt/ -catenin and Bmp signals control distinct sets of transcription factors in cardiac progenitor cells. Proc. Natl. Acad. Sci. 109, 10921-10926.

Lawson, K. A., Meneses, J. J. and Pedersen, R. A. (1991). Clonal analysis of epiblast fate during germ layer formation in the mouse embryo. Development 113, 891-911.

Lee, J. H., Protze, S. I., Laksman, Z., Backx, P. H. and Keller, G. M. (2017). Human Pluripotent Stem Cell-Derived Atrial and Ventricular Cardiomyocytes Develop from Distinct Mesoderm Populations. Cell Stem Cell 21, 179-194.e4.

Lescroart, F., Chabab, S., Lin, X., Rulands, S., Paulissen, C., Rodolosse, A., Auer, H., Achouri, Y., Dubois, C., Bondue, A., et al. (2014). Early lineage restriction in temporally distinct populations of Mesp1 progenitors during mammalian heart development. Nat. Cell Biol. 16, 829-840.

Lescroart, F., Wang, X., Lin, X., Swedlund, B., Gargouri, S., Sànchez-Dànes, A., Moignard, V., Dubois, C., Paulissen, C., Kinston, S., et al. (2018). Defining the earliest step of cardiovascular lineage segregation by single-cell RNA-seq. Science (80-. ). 359, 1177-1181.

Lian, X., Zhang, J., Azarin, S. M., Zhu, K., Hazeltine, L. B., Bao, X., Hsiao, C., Kamp, T. J. and Palecek, S. P. (2013). Directed cardiomyocyte differentiation from human pluripotent stem cells by modulating $W n t / \beta$-catenin signaling under fully defined conditions. Nat. Protoc. 8, 162-75.
Love, M. I., Huber, W. and Anders, S. (2014). Moderated estimation of fold change and dispersion for RNA-seq data with DESeq2. Genome Biol. 15, 550.

Marvin, M. J., Di Rocco, G., Gardiner, A., Bush, S. M. and Lassar, A. B. (2001). Inhibition of Wnt activity induces heart formation from posterior mesoderm. Genes Dev. 15, 316-327.

Meilhac, S. M., Lescroart, F., Blanpain, C. D. and Buckingham, M. E. (2014). Cardiac cell lineages that form the heart. Cold Spring Harb. Perspect. Med. 4, a013888.

Miazga, C. M. and McLaughlin, K. A. (2009). Coordinating the timing of cardiac precursor development during gastrulation: A new role for Notch signaling. Dev. Biol. 333, 285-296.

Morohashi, Y., Kan, T., Tominari, Y., Fuwa, H., Okamura, Y., Watanabe, N., Sato, C., Natsugari, H., Fukuyama, T., Iwatsubo, T., et al. (2006). C-terminal fragment of presenilin is the molecular target of a dipeptidic $\mathrm{Y}$-secretase-specific inhibitor DAPT (N-[N-(3,5-difluorophenacetyl)-L- alanyl]-S-phenylglycine t-butyl ester). J. Biol. Chem. 281, 14670-14676.

Nascone, N. and Mercola, M. (1995). An inductive role for the endoderm in Xenopus cardiogenesis. Development 121, 515-23.

Saga, Y., Miyagawa-Tomita, S., Takagi, A., Kitajima, S., Miyazaki, J. i and Inoue, T. (1999). MesP1 is expressed in the heart precursor cells and required for the formation of a single heart tube. Development 126, 3437-3447.

Schneider, V. A. and Mercola, M. (2001). Wnt antagonism initiates cardiogenesis in Xenopus laevis. Genes Dev. 15, 304-315.

Schultheiss, T. ., Xydas, S. and Lassar, A. . (1995). Induction of avian cardiac myogenesis by anterior endoderm. Development 121, 4203-4214.

Scialdone, A., Tanaka, Y., Jawaid, W., Moignard, V., Wilson, N. K., Macaulay, I. C., Marioni, J. C. 
and Göttgens, B. (2016). Resolving early mesoderm diversification through single-cell expression profiling. Nature 535, 289-293.

Skelton, R. J. P., Brady, B., Khoja, S., Sahoo, D., Engel, J., Arasaratnam, D., Saleh, K. K., Abilez, O. J., Zhao, P., Stanley, E. G., et al. (2016). CD13 and ROR2 Permit Isolation of Highly Enriched Cardiac Mesoderm from Differentiating Human Embryonic Stem Cells. Stem Cell Reports 6, 95-108.

Souilhol, C., Perea-Gomez, A., Camus, A., Beck-Cormier, S., Vandormael-Pournin, S., Escande, M., Collignon, J. and Cohen-Tannoudji, M. (2015). NOTCH activation interferes with cell fate specification in the gastrulating mouse embryo. Development 142, 3649-3660.

Takakura, N., Yoshida, H., Ogura, Y., Kataoka, H., Nishikawa, S. and Nishikawa, S. I. (1997). PDGFRa expression during mouse embryogenesis: Immunolocalization analyzed by whole-mount immunohistostaining using the monoclonal anti-mouse PDGFRa antibody APA5. J. Histochem. Cytochem. 45, 883-893.

Tam, P. P., Parameswaran, M., Kinder, S. J. and Weinberger, R. P. (1997). The allocation of epiblast cells to the embryonic heart and other mesodermal lineages: the role of ingression and tissue movement during gastrulation. Development 124, 1631-42.

Vincent, S. D. and Buckingham, M. E. (2010). How to make a heart. The origin and regulation of cardiac progenitor cells, in: Current Topics in Developmental Biology. https://doi.org/10.1016/S00702153(10)90001-X

Wang, Y., Lu, P., Wu, B., Morrow, B. E. and Zhou, B. (2018). NOTCH maintains developmental cardiac gene network through WNT5A. J. Mol. Cell. Cardiol. 125, 98-105.

Watanabe, Y. (2006). Activation of Notch1 signaling in cardiogenic mesoderm induces abnormal heart morphogenesis in mouse. Development 133, 1625-1634.

Xavier-Neto, J., Neville, C. M., Shapiro, M. D.,
Houghton, L., Wang, G. F., Nikovits, W., Stockdale, F. E. and Rosenthal, N. (1999). A retinoic acid-inducible transgenic marker of sino-atrial development in the mouse heart. Development 126, 2677-87.

Yang, L., Soonpaa, M. H., Adler, E. D., Roepke, T. K., Kattman, S. J., Kennedy, M., Henckaerts, E., Bonham, K., Abbott, G. W., Linden, R. M., et al. (2008). Human cardiovascular progenitor cells develop from a KDR+ embryonic-stem-cell-derived population. Nature 453, 524-528.

Yutzey, K. E. and Bader, D. (1995). Diversification of cardiomyogenic cell lineages during early heart development. Circ. Res. 77, 216-219. 
bioRxiv preprint doi: https://doi.org/10.1101/2020.02.20.958348; this version posted February 20, 2020. The copyright holder for this preprint (which was not certified by peer review) is the author/funder, who has granted bioRxiv a license to display the preprint in perpetuity. It is made available under aCC-BY-NC-ND 4.0 International license.

a.

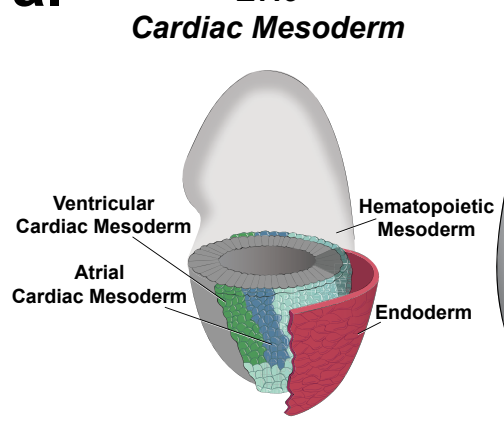

E8.25 Cardiac Crescent

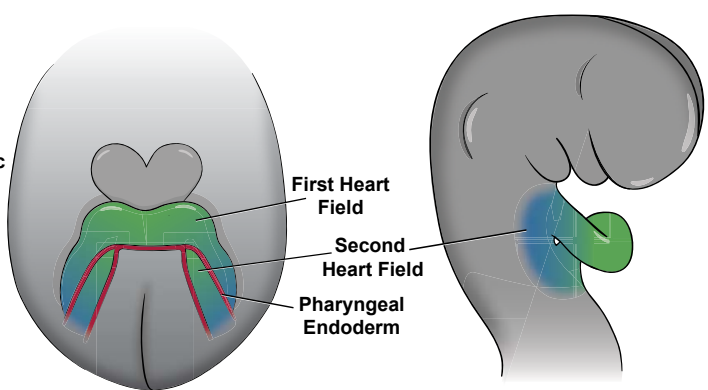

E8.5 Early Heart Tube
E15.5 Four-chambered Heart

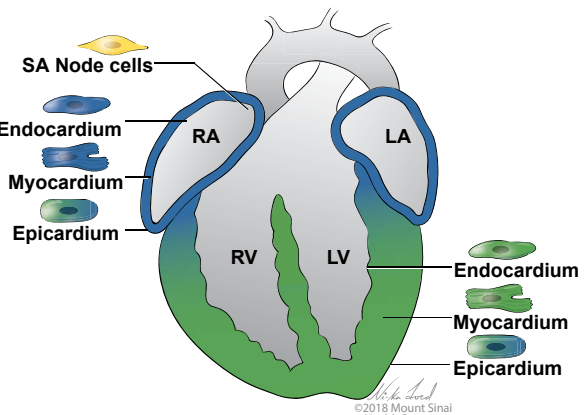

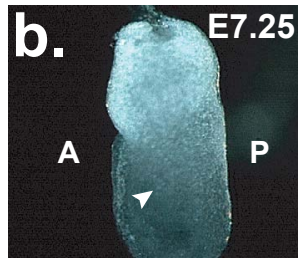

Brightfield

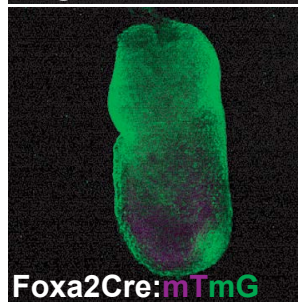

Foxa2Cre:me
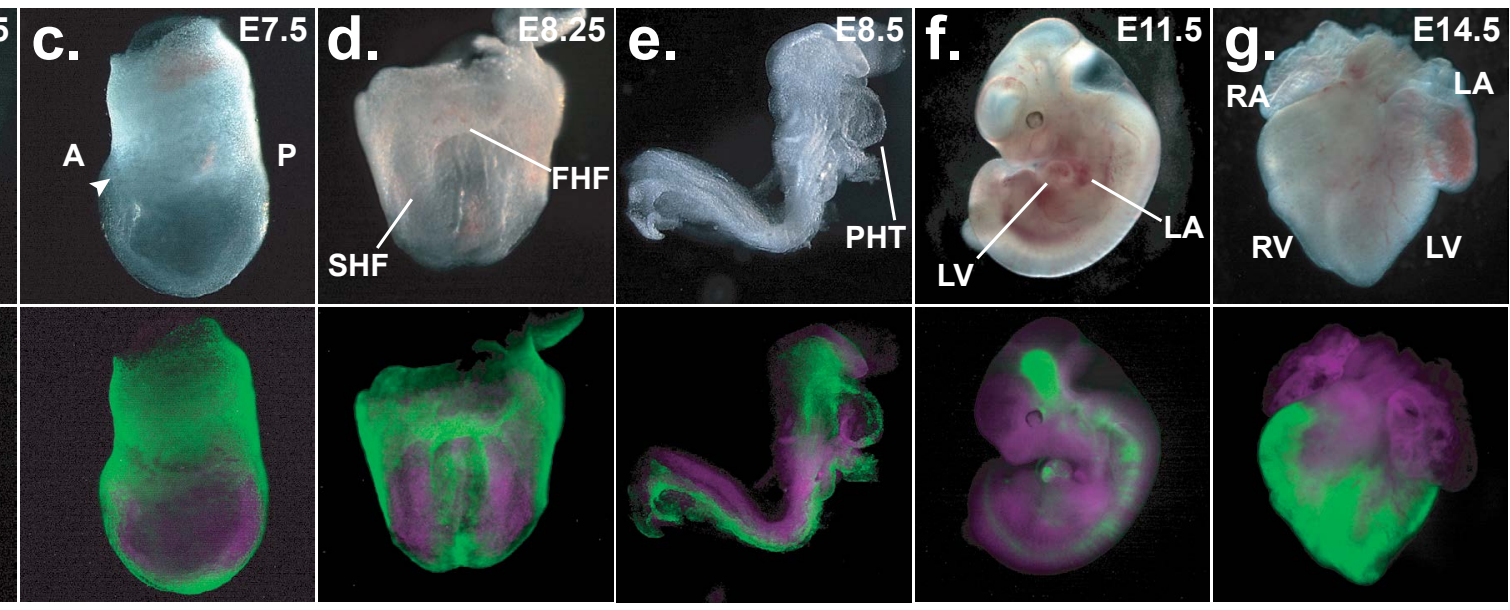

Supplementary Figure 1: Distribution of Foxa2Cre:YFP lineage-traced cells during mouse development. (a) Schematic overview of ventricular progenitors during heart development. (b-g) Whole mount live imaging of Foxa2Cre:mTmG lineage tracing embryos. Early (b) and late (c) gastrulation stages show GFP+ migratory mesoderm. Cardiac crescent (d) and early heart tube (e) stage embryos show restriction of GFP+ cells to the prospective ventricular regions. GFP+ cells are restricted to the ventricles in E11.5 (f) and E14.5 (g) hearts. 


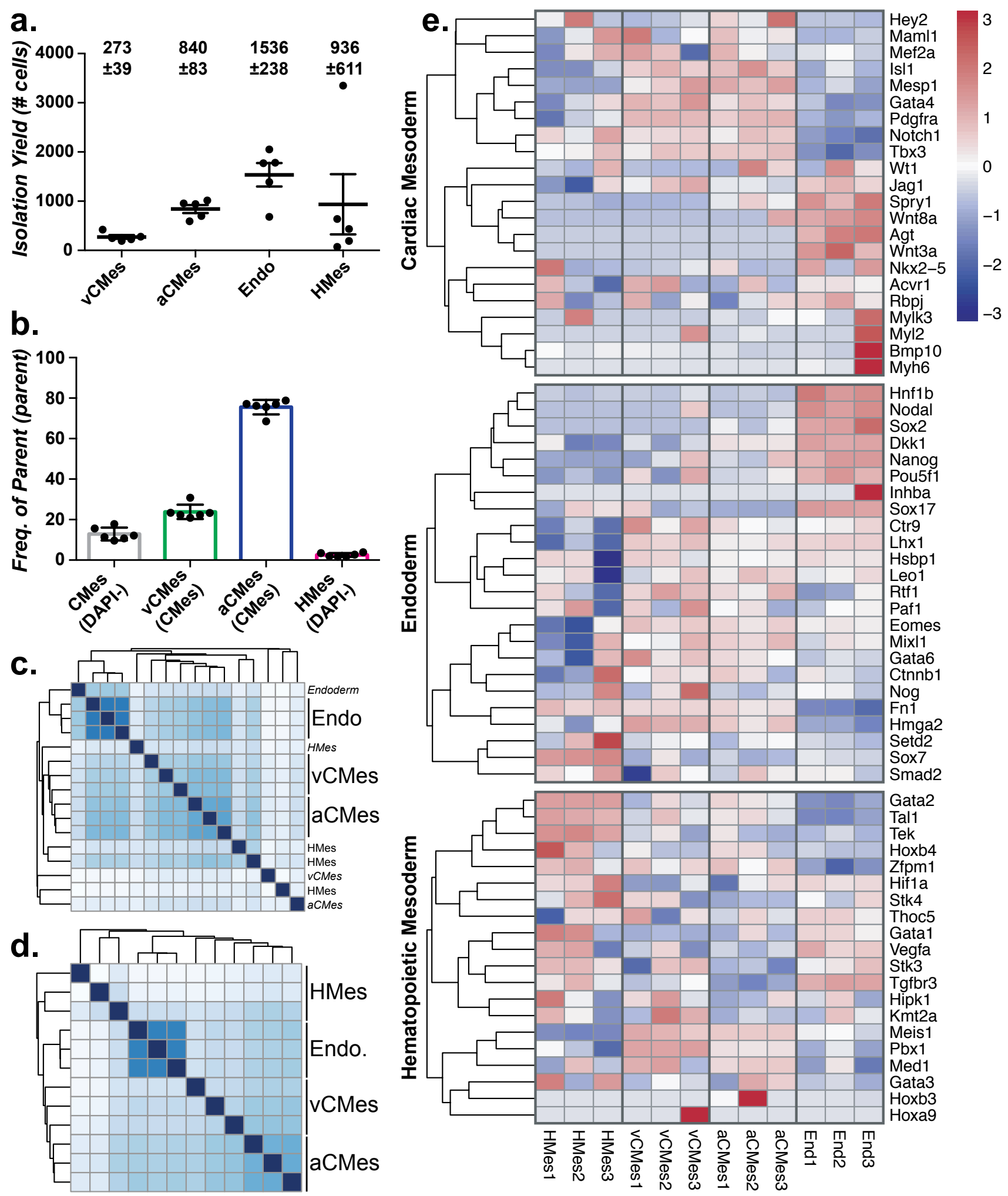

Supplementary Figure 2: Isolation and gene expression profiling of embryonic progenitor populations. (a) Cell yield from FACS isolation experiments. E7.5 mouse embryos were stage-matched by morphology and pooled by litter. Cell numbers are for pools of 7-10 embryos. (b) Cardiac mesoderm contributed to approximately $10 \%$ of total viable cells. Ventricular cardiac mesoderm made up a smaller portion of total cardiac mesoderm than non-ventricular cardiac mesoderm. Hematopoietic mesoderm made up $\sim 2.5 \%$ of the total viable population. (c, d) A total of four replicates were subjected to RNA sequencing and analyzed by hierarchical clustering. Based on this analysis, one replicate was removed prior to further analysis. (e) Expression z-scores for cardiac mesoderm, endoderm, and hematopoietic mesoderm signature genes. 

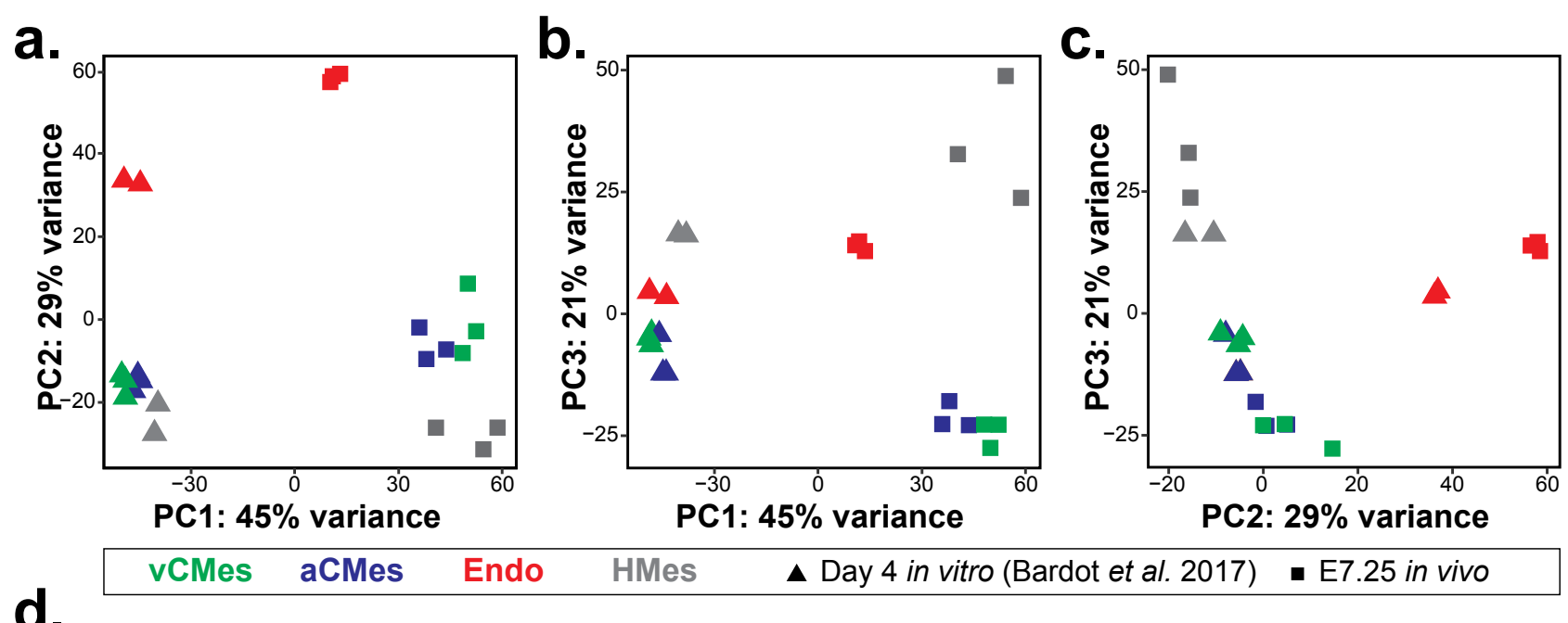

\begin{tabular}{|c|c|c|c|c|c|}
\hline PC1- (in vitro) & PC1+ (in vivo) & PC2-(Mes) & PC2+ (Endo.) & PC3-(CMes) & PC3+ (HMes/E) \\
\hline Mmp9 & Xist & Tal1 & Pkd1/1 & Pcdh19 & 1700082M22Rik \\
\hline KIhl26 & B830012L14Rik & Unc5c & Ermn & Foxf1 & Timm8a2 \\
\hline Gm13225 & C79798 & Gata2 & Cer1 & D430041D05Rik & Nrip3 \\
\hline Arrdc2 & 4933426K07Rik & Fli1 & Irs4 & Fendrr & Gm8200 \\
\hline Jak3 & Gm26749 & Hapln1 & Miat & Sh3rf3 & Ikzf1 \\
\hline Arhgap36 & Gm15982 & $\mathrm{Kdr}$ & Foxd3 & Pdgfra & Cdh5 \\
\hline Dact2 & Gm6211 & Cdh5 & Enpp3 & Cacna1c & Sox7 \\
\hline Capg & Fth-ps2 & Pcdh12 & Eras & Lefty2 & Emx2 \\
\hline Aacs & Gm11935 & Fgf3 & Six3 & Snai1 & Clic6 \\
\hline Pear1 & Gm20407 & Ptprb & 2610528J11Rik & L1cam & Lcp1 \\
\hline Slc16a13 & Gm9930 & Gpr124 & Foxj1 & Slitrk2 & Myb \\
\hline Gale & Gm16552 & Myzap & Hnf4a & Mesp1 & Tek \\
\hline Kif1a & Gm13854 & Erg & Samd3 & Pmepa1 & Slc7a8 \\
\hline Lipg & Gm16121 & Gadd45g & Apob & Hand2 & Scube2 \\
\hline Zbtb7b & Gm26603 & Mmp9 & Dtna & Gm15413 & Slc32a1 \\
\hline Ntrk1 & Gm26625 & Etv2 & Pilra & Wnt5a & Fam65b \\
\hline Lmtk3 & Grb10 & Scube2 & Hnf1b & DII1 & Maf \\
\hline Pnpla3 & Gm15414 & Tek & Ntn1 & Tbx5 & F2rl1 \\
\hline Aldh1/1 & B3galt2 & Rhoj & Cdh1 & Fignl2 & Gm14204 \\
\hline Alox15 & KCNQ1OT1_5 & Eng & Slc34a2 & Srrm4 & Wnk4 \\
\hline Stub1 & Gm26580 & Gypc & Rbm47 & Rimbp2 & Gata1 \\
\hline Sgsm1 & AU040972 & Pcsk5 & Foxa2 & Scn4a & $n-R 5 s 201$ \\
\hline Pdgfrl & 9230105E05Rik & Wnt2 & Chrd & Fbln2 & Slc1a3 \\
\hline Sh2d3c & Lrrtm2 & Hoxd1 & Apoa1 & Meis2 & Itgb3 \\
\hline Brf2 & Gm14540 & Fam212a & Pga5 & 1700006J14Rik & Gm16110 \\
\hline Zscan25 & Hist1h1e & Sox7 & Hkdc1 & $n-R 5 s 54$ & Tmem240 \\
\hline Gcat & Hist1h4d & Pear1 & Mia2 & Tdo2 & Gfi1b \\
\hline Uap1/1 & Hist1h2bm & $\mathrm{HIx}$ & Foxa1 & Meis1 & Tie1 \\
\hline Gsc & Filip1I & Nrip3 & Spef2 & Ntng1 & Ddah1 \\
\hline Sgsm2 & Gm26789 & Foxf1 & Wdr52 & Zcchc12 & Oit3 \\
\hline
\end{tabular}

Supplementary Figure 3: Comparison of RNA sequencing data from in vitro and in vivo populations of interest. (a) Principal component analysis of in vitro and in vivo cardiac mesoderm, endoderm, and hematopoietic mesoderm RNA sequencing data. The first three PCs capture $95 \%$ of the total variance across all samples. (a, b) PC1 separates samples based on cell origin and technical differences (in vitro vs. in vivo). (a) PC2 separates endoderm from mesodermal cell types, whereas (b) PC3 separates cardiac mesoderm from non-cardiac samples. (c) Comparison of PC2 vs. PC3 restores the lineage relationships observed when analyzing within in vitro or in vivo datasets. (d) Ordered gene lists for genes that explain variance within each principal component. 

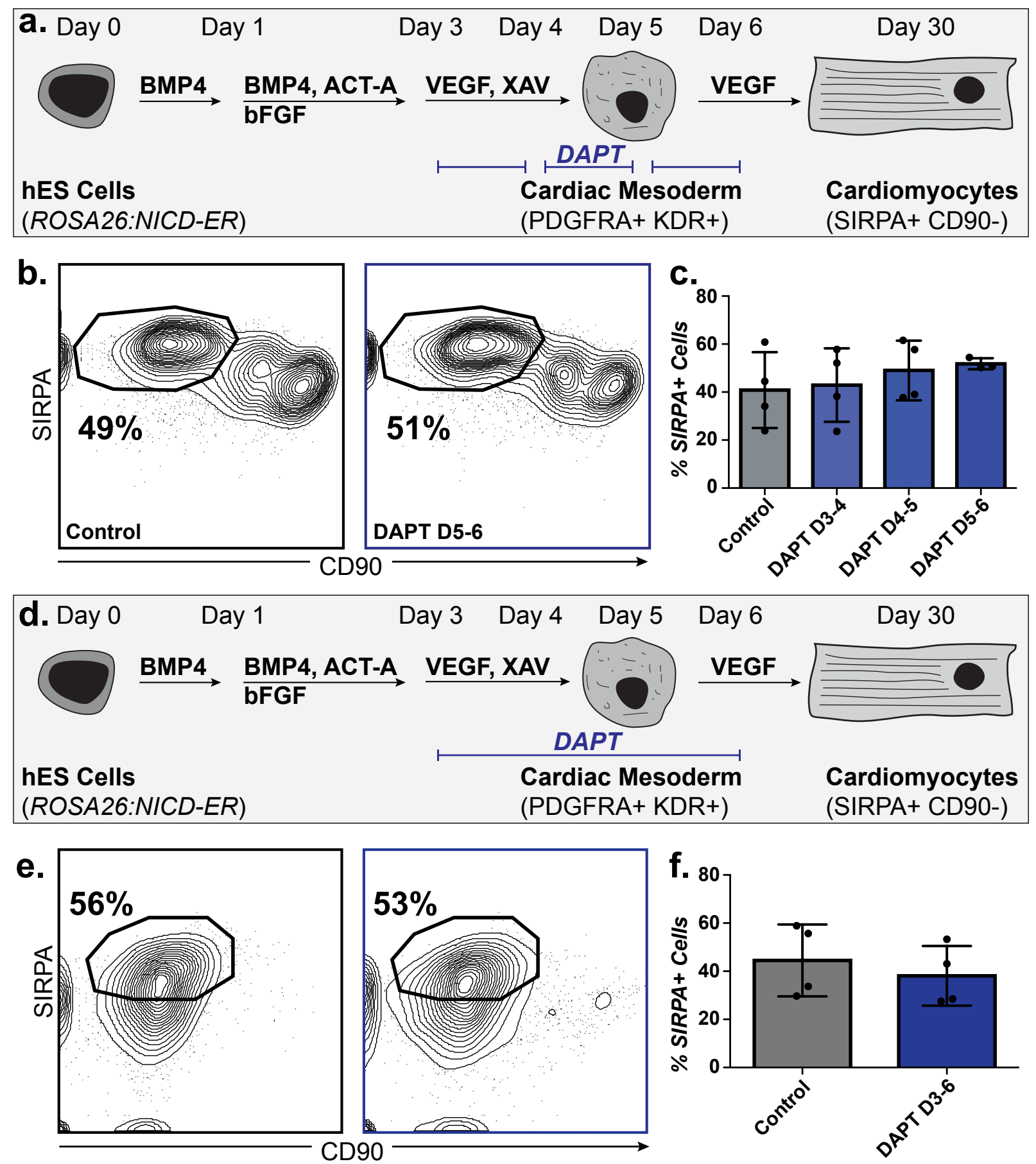

Supplementary Figure 4: Inhibition of Notch has no effect on directed differentiation of cardiomyocytes from hPSCs. (a) Schematic of ROSA26:NICD-ER hPSC-cardiomyocyte differentiation and Notch inhibition times. (b) Flow cytometric analysis with antibodies against SIRPA and CD90 to measure cardiomyocyte (SIRPA+CD90-) differentiation efficiency at day 30 in control cultures (left) or after DAPT treatment from day 5-6 (right). (c) Quantification of data in (b). (d) Schematic of MYL2-GFP hiPSC-cardiomyocyte differentiation and Notch inhibition times. (e) Flow cytometric analysis with antibodies against SIRPA and CD90 to measure cardiomyocyte (SIRPA+CD90-) differentiation efficiency at day 30 in control cultures (left) or after DAPT treatment from day 3-6 (right). (f) Quantification of data in (e). 

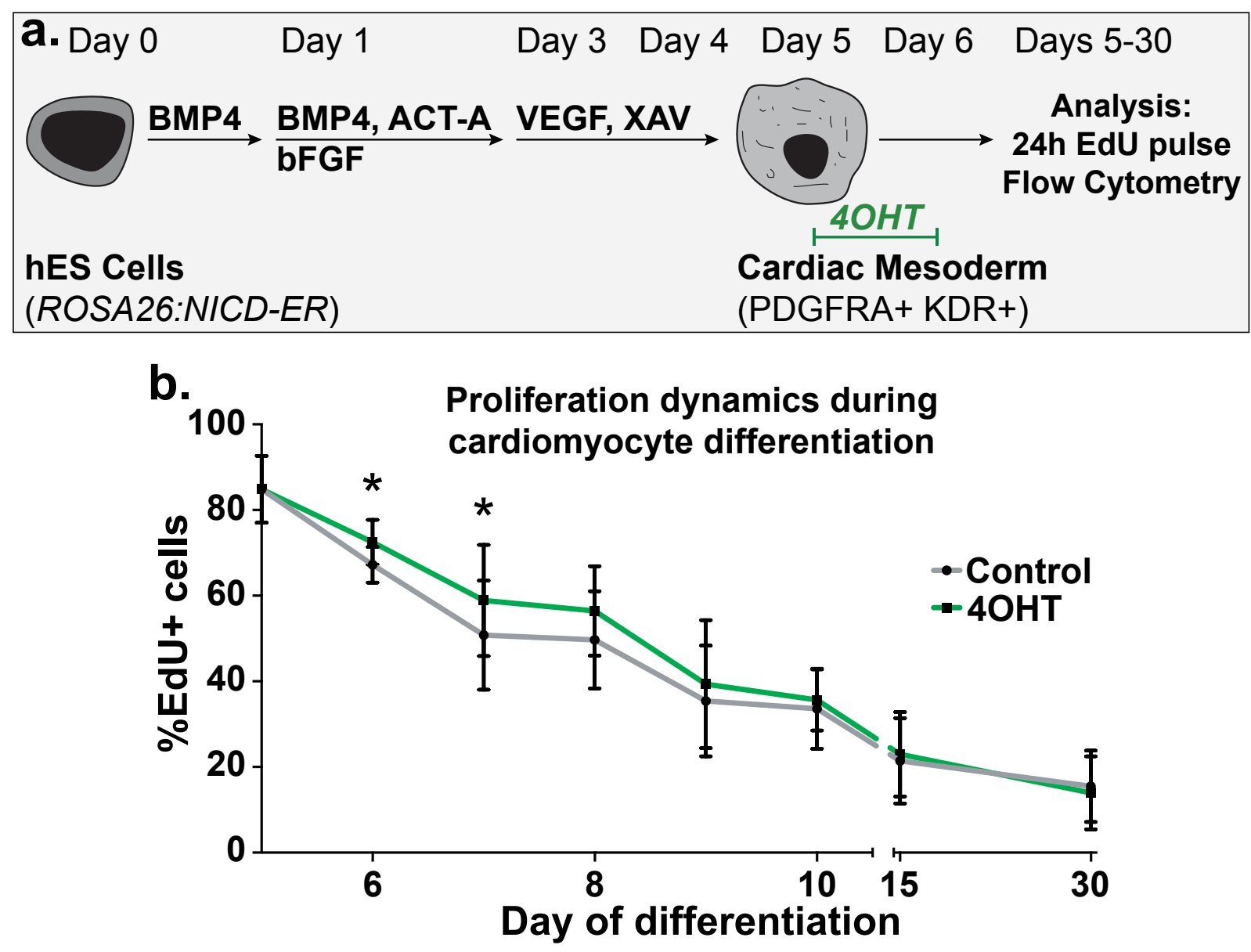

Supplementary Figure 5: Notch induction briefly extends the proliferative window of cardiac precursors during hPSC directed differentiation. (a) Schematic of ROSA26:NICD-ER hPSC-cardiomyocyte differentiation and EdU analysis. Cultures were either untreated or treated with $4 \mathrm{OHT}$ from day 5-6 to induce Notch signaling. EdU was added $24 \mathrm{~h}$ before each collection and analysis time. (b) EdU incorporation analysis by flow cytometry from day 5 to day 30 in control or Notch-induced cultures ( $\mathrm{n}=5$ differentiations). 

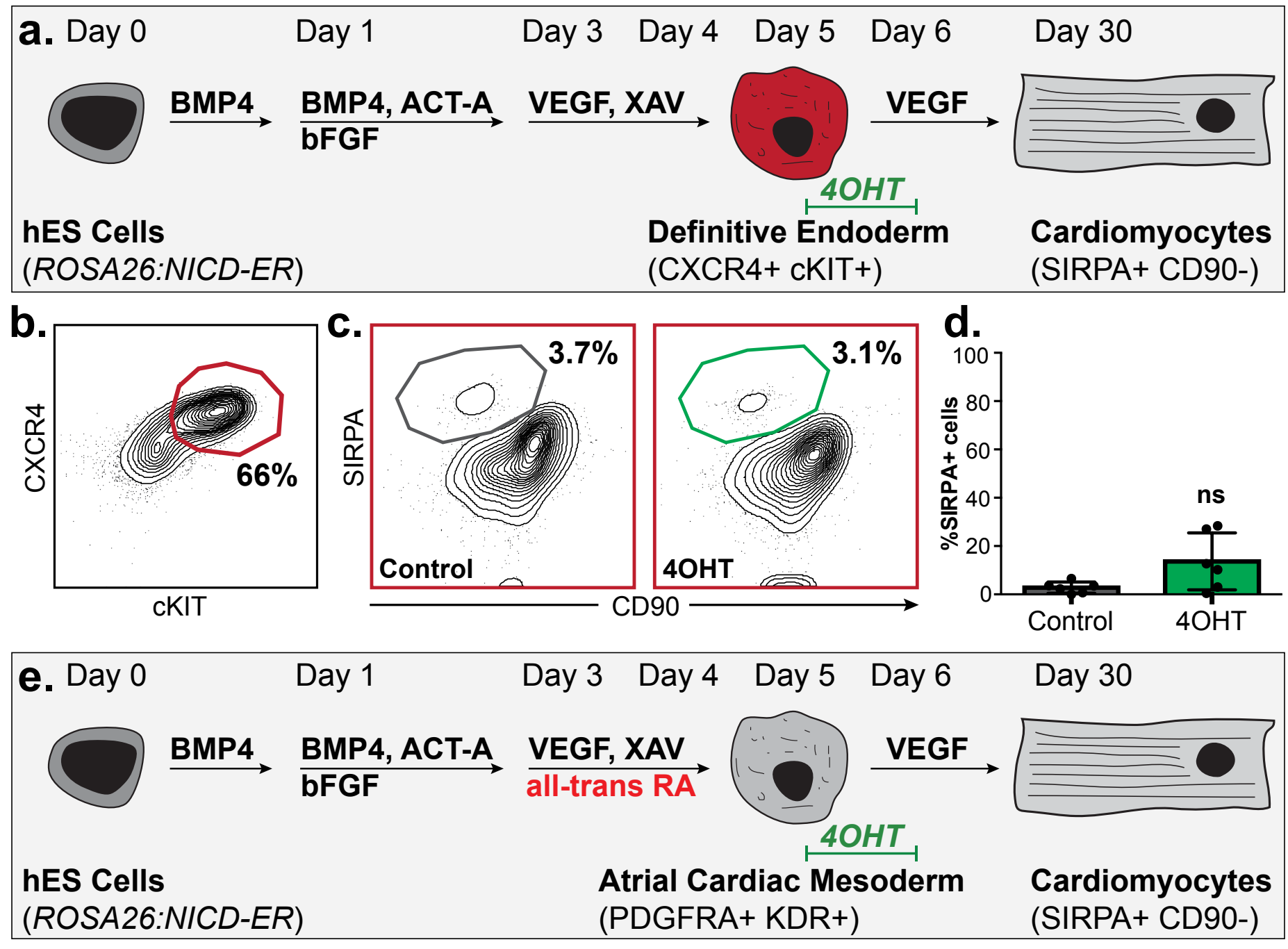

f.
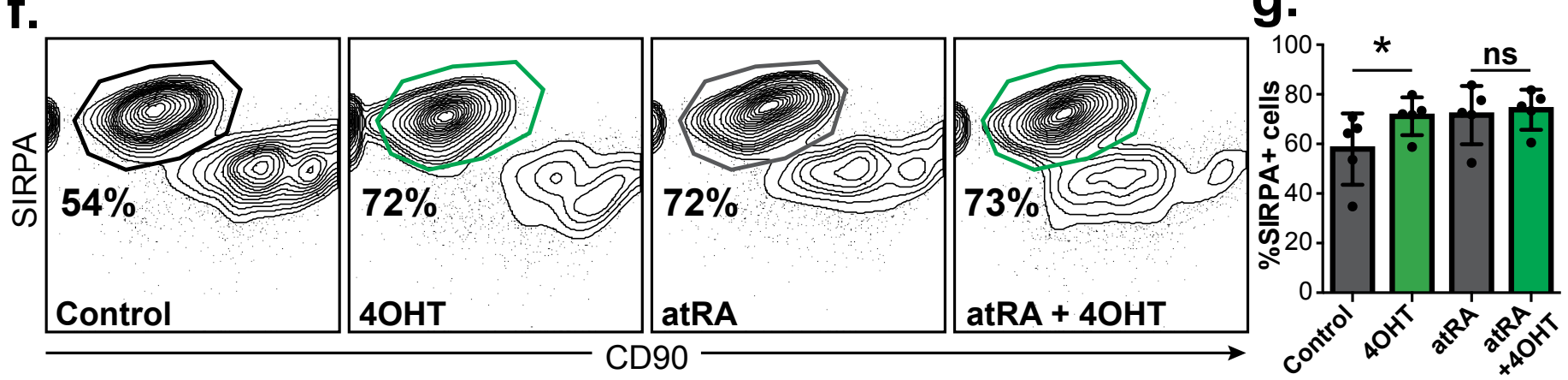

Supplementary Figure 6: Induction of Notch signaling does not induce cardiomyocyte differentiation from definitive endoderm progenitors. (a) Schematic of ROSA26:NICD-ER hPSC differentiation to definitive endoderm progenitors, and continued differentiation in basal media. (b) Flow cytometric analysis of definitive endoderm progenitor generation with antibodies against CXCR4 and CKIT. (c) Flow cytometric analysis of cardiomyocyte generation from definitive endoderm cultured in basal media and the absence or presence of Notch induction from day 5-6. (d) Quantification of data in (c). (e) Schematic of ROSA26:NICD-ER hPSC differentiation to atrial cardiomyocytes through addition of all-trans retinoic acid (atRA). (f) Flow cytometric analysis of cardiomyocyte differentiation efficiency from ventricular (control) or atrial (atRA) cultures in the absence or presence of Notch induction from day 5-6. (g) Quantification of data in (f). 


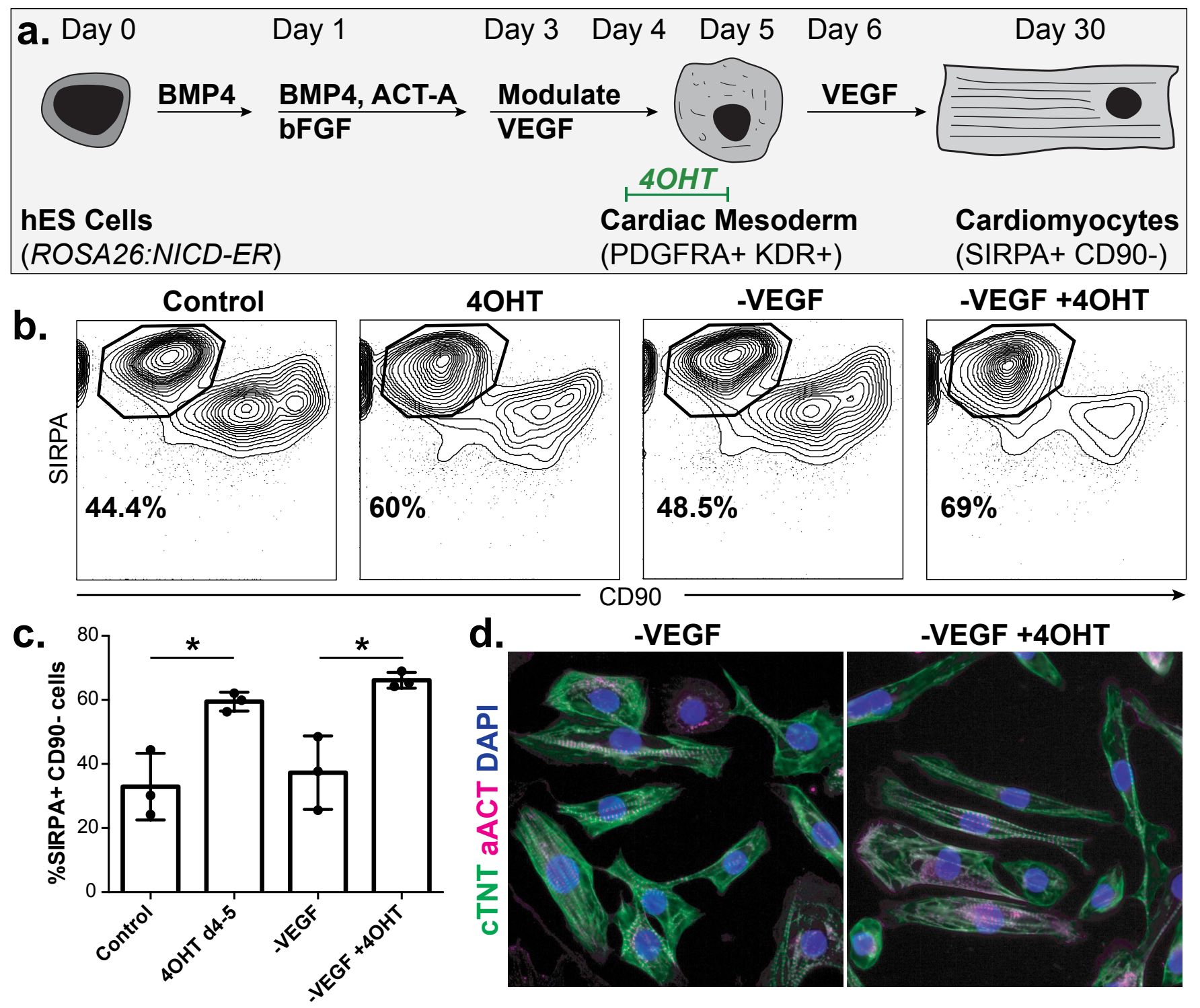

Supplementary Figure 7: VEGF is not required for cardiomyocyte differentiation efficiency. (a) Schematic of ROSA26:NICD-ER hPSC-cardiomyocyte differentiation with WNT and VEGF modulation and Notch induction. (b) Flow cytometric analysis of cardiomyocyte generation in the absence of VEGF or WNT and VEGF, with and without Notch induction. (c) Quantification of data in (b). (d) Immunofluorescence analysis on day 30 cardiomyocytes with antibodies against cTNT and aACT. 